\title{
Plattenbauten: Touching Rectangles in Space ${ }^{\star}$
}

\author{
Stefan Felsner ${ }^{1}$, Kolja Knauer ${ }^{2}$, and Torsten Ueckerdt ${ }^{3}$ \\ 1 Institute of Mathematics, Technische Universität Berlin (TUB), Berlin, Germany \\ 2 Departament de Matemàtiques i Informàtica, Universitat de Barcelona (UB), \\ Barcelona, Spain \\ Aix-Marseille Univ, Université de Toulon, CNRS, LIS, Marseille, France \\ 3 Institute for Theoretical Informatics, Karlsruhe Institute of Technology (KIT), \\ Karlsruhe, Germany
}

\begin{abstract}
Planar bipartite graphs can be represented as touching graphs of horizontal and vertical segments in $\mathbb{R}^{2}$. We study a generalization in space, namely, touching graphs of axis-aligned rectangles in $\mathbb{R}^{3}$. We prove that planar 3-colorable graphs can be represented as touching graphs of axis-aligned rectangles in $\mathbb{R}^{3}$. The result implies a characterization of corner polytopes previously obtained by Eppstein and Mumford. A by-product of our proof is a distributive lattice structure on the set of orthogonal surfaces with given skeleton.

Moreover, we study the subclass of strong representations, i.e., families of axis-aligned rectangles in $\mathbb{R}^{3}$ in general position such that all regions bounded by the rectangles are boxes. We show that the resulting graphs correspond to octahedrations of an octahedron. This generalizes the correspondence between planar quadrangulations and families of horizontal and vertical segments in $\mathbb{R}^{2}$ with the property that all regions are rectangles.
\end{abstract}

Keywords: Touching graphs, contact graphs, boxicity, planar graphs

\section{Introduction}

The importance of contact and intersection representations of graphs stems not only from their numerous applications including information visualization, chip design, bio informatics and robot motion planning (see for example the references in 2,10 ), but also from the structural and algorithmic insights accompanying the investigation of these intriguing geometric arrangements. From a structural point of view, the certainly most fruitful contact representations (besides the "Kissing Coins" of Koebe, Andrew, and Thurston [1, 17, 24]) are axis-aligned segment contact representations: families of interior-disjoint horizontal and vertical segments in $\mathbb{R}^{2}$ where the intersection of any two segments is either empty or an endpoint of at least one of the segments. The corresponding touching

\footnotetext{
* An extended abstract appears in the proceedings of the 46th International Workshop on Graph-Theoretic Concepts in Computer Science (WG 2020).
} 
graph ${ }^{4}$ has the segments as its vertices and the pairs of segments as its edges for which an endpoint of one segment is an interior point of the other segment, see the left of Fig. 1. It has been discovered several times 16,22 that any such touching graph is bipartite and planar, and that these two obviously necessary conditions are in fact already sufficient: Every planar bipartite graph is the touching graph of interior-disjoint axis-aligned segments in $\mathbb{R}^{2}$. In fact, edgemaximal segment contact representations endow their associated plane graphs with many useful combinatorial structures such as 2-orientations [10], separating decompositions 4, bipolar orientations 23, 25], transversal structures [13, and Schnyder woods [27].
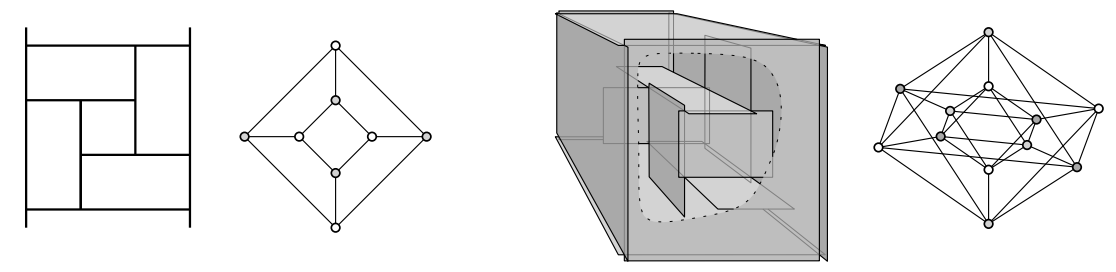

Fig. 1. An axis-aligned segment contact representation (left) and a Plattenbau (right) together with the respective touching graphs.

In this paper we extend axis-aligned segment contact representations in $\mathbb{R}^{2}$ to axis-aligned rectangle contact representations in $\mathbb{R}^{3}$. That is, we consider families $\mathcal{R}$ of axis-aligned closed and bounded rectangles in $\mathbb{R}^{3}$ with the property that for all $R, R^{\prime} \in \mathcal{R}$ the intersection $R \cap R^{\prime}$ is a subset of the boundary of at least one of them, i.e., the rectangles are interiorly disjoint. We call such a family a Plattenbay 5 . Given a Plattenbau $\mathcal{R}$ one can consider its intersection graph $I_{\mathcal{R}}$. However, for us the more important concept is a certain subgraph of $I_{\mathcal{R}}$, called the touching graph $G_{\mathcal{R}}$ of $\mathcal{R}$. There is one vertex in $G_{\mathcal{R}}$ for each rectangle in $\mathcal{R}$ and two vertices are adjacent if the corresponding rectangles touch, i.e., their intersection is non-empty and contains interior points of one and only one of the rectangles. We say that $G$ is a Plattenbau graph if there is a Plattenbau $\mathcal{R}$ such that $G \cong G_{\mathcal{R}}$. In this case we call $\mathcal{R}$ a Plattenbau representation of $G$.

Plattenbauten are a natural generalization of axis-aligned segment contact representations in $\mathbb{R}^{2}$ and thus Plattenbau graphs are a natural generalization of planar bipartite graphs. While clearly all Plattenbau graphs are tripartite (properly vertex 3 -colorable), it is an interesting challenge to determine the exact topological properties in $\mathbb{R}^{3}$ that hold for all Plattenbau graphs, thus generalizing the

\footnotetext{
${ }^{4}$ We use the term touching graphs rather than the more standard contact graph to underline the fact that segments with coinciding endpoints (e.g., two horizontal segments touching a vertical segment in the same point but from different sides, but also non-parallel segments with coinciding endpoint) do not form an edge.

${ }^{5}$ Plattenbau (plural Plattenbauten) is a German word describing a building (Bau) made of prefabricated concrete panels (Platte).
} 
concept of planarity from 2 to 3 dimensions (for tripartite graphs). We present results towards a characterization of Plattenbau graphs in three directions.

Our Results and Organization of the Paper. In Section 2 we provide some simple examples of Plattenbau graphs and give some simple necessary conditions for all Plattenbau graphs. We observe that unlike touching graphs of segments, general Plattenbau graphs are not closed under taking subgraphs. We circumvent this issue by introducing the subclass of proper Plattenbauten where for any two touching rectangles $R, R^{\prime}$ the intersection $R \cap R^{\prime}$ must be a boundary edge of one of $R, R^{\prime}$. Moreover, we introduce boxed Plattenbauten where every bounded region of $\mathbb{R}^{3}$ is a box, and discuss questions of augmentability.

In Section 3 we show that within planar graphs the necessary condition of 3-colorability is also sufficient for Plattenbau graphs. Thus, the topological characterization of Plattenbau graphs must fully contain planarity (which is not obvious as we consider 3 -colorable graphs and not only bipartite graphs).

Theorem 1. Every 3-colorable planar graph is the touching graph of a proper Plattenbau.

Along the proof of Theorem 1, we obtain a characterization of skeletons of orthogonal surfaces which is implicit already in work of Eppstein and Mumford 6. Another proof of Theorem 1 can be obtained from Gonçalves' recent proof that 3-colorable planar graphs admit segment intersection representations with segments of 3 slopes 14 . We further comment on these alternative approaches in Section 3.3. A consequence of our approach is a natural partial order - namely a distributive lattice - on the set of orthogonal surfaces with a given skeleton.

In Section 4 we consider proper and boxed Plattenbau graphs as the 3dimensional correspondence to the edge-maximal planar bipartite graphs, the quadrangulations. We give a complete characterization of these graphs.

Theorem 2. A graph $G$ is the touching graph of a proper boxed Plattenbau $\mathcal{R}$ if and only if there are six outer vertices in $G$ such that each of the following holds:

(P1) $G$ is connected and the outer vertices of $G$ induce an octahedron.

(P2) The edges of $G$ admit an orientation such that

- the bidirected edges are exactly the outer edges,

- each vertex has exactly 4 outgoing edges.

(P3) The neighborhood $N(v)$ of each vertex $v$ induces a spherical quadrangulation $S Q(v)$ in which the out-neighbors of $v$ induce a 4-cycle.

- If $v$ is an outer vertex, this 4-cycle bounds a face of $S Q(v)$.

(P4) For every edge uv of $G$ with common neighborhood $C=N(u) \cap N(v)$, the cyclic ordering of $C$ around $u$ in $S Q(v)$ is the reverse of the cyclic ordering of $C$ around $v$ in $S Q(u)$. 
A spherical quadrangulation is a graph embedded on the 2-dimensional sphere without crossings with all faces bounded by a 4-cycle. Spherical quadrangulations are 2-connected, planar, and bipartite. We remark that Theorem 2 does not give a complete characterization of proper Plattenbau graphs since some proper Plattenbau graphs are not contained in any proper boxed Plattenbau graph as discussed in Section 2.

\section{Types of Plattenbauten and Questions of Augmentation}

Let us observe some properties of Plattenbau graphs. Clearly, the class of all Plattenbau graphs is closed under taking induced subgraphs. Examples of Plattenbau graphs are $K_{2,2, n}$, see Fig. 2, and the class of grid intersection graphs, i.e., bipartite intersection graphs of axis-aligned segments in the plane [16]. For the latter take the segment intersection representation of a graph, embed it into the $x y$-plane in $\mathbb{R}^{3}$ and thicken all horizontal segments a small amount into $y$ direction and all vertical segments a bit into $z$-direction outwards the $x y$-plane. In particular, $K_{m, n}$ is a Plattenbau graph, see Fig. 2. In order to exclude some graphs, we observe some necessary properties of all Plattenbau graphs.
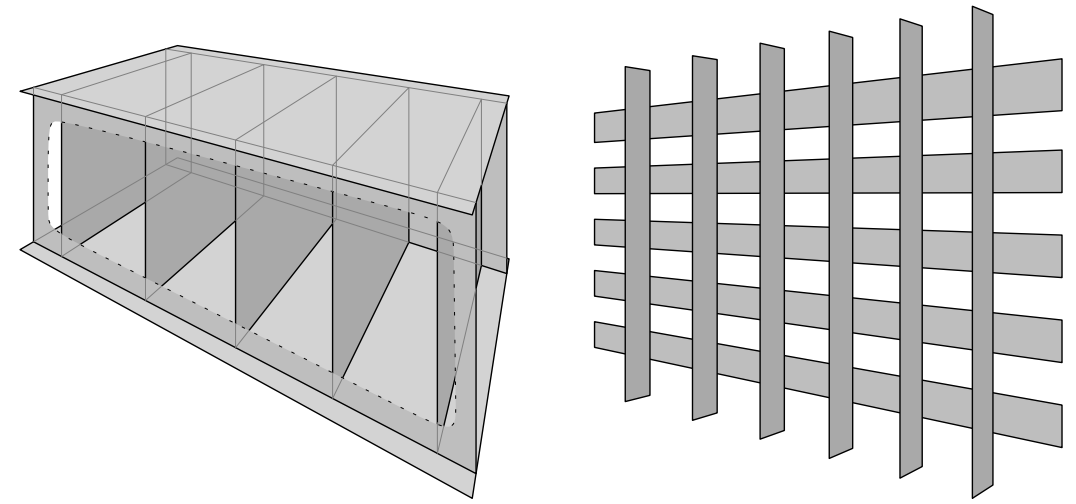

Fig. 2. Plattenbau representations of $K_{2,2,5}$ (left) and $K_{5,6}$ (right).

Observation 1 If $G$ is a Plattenbau graph, then

1. the chromatic number of $G$ is at most 3 ,

2. the neighborhood of any vertex of $G$ is planar.

3. the boxicity of $G$, i.e., the smallest dimension $d$ such that $G$ is the intersection graph of boxes in $\mathbb{R}^{d}$, is at most 3 .

Proof. Item 1: Each orientation class is an independent set.

Item 2. Let $v$ be a vertex of $G$ represented by $R \in \mathcal{R}$. Let $H$ be the supporting hyperplane of $R$ and $H^{+}, H^{-}$the corresponding closed halfspaces. The 
neighborhood $N(v)$ consists of rectangles $R^{+}$intersecting $H^{+}$and those $R^{-}$intersecting $H^{-}$. The rectangles in each of these sets have a plane touching graph, since it corresponds to the touching graph of the axis-aligned segments given by their intersections with $R$. The neighboring rectangles in $R^{+} \cap R^{-}$are on the outer face in both graphs in opposite order, so identifying them gives a planar drawing of the graph induced by $N(v)$. See Fig. 3 for an illustration.
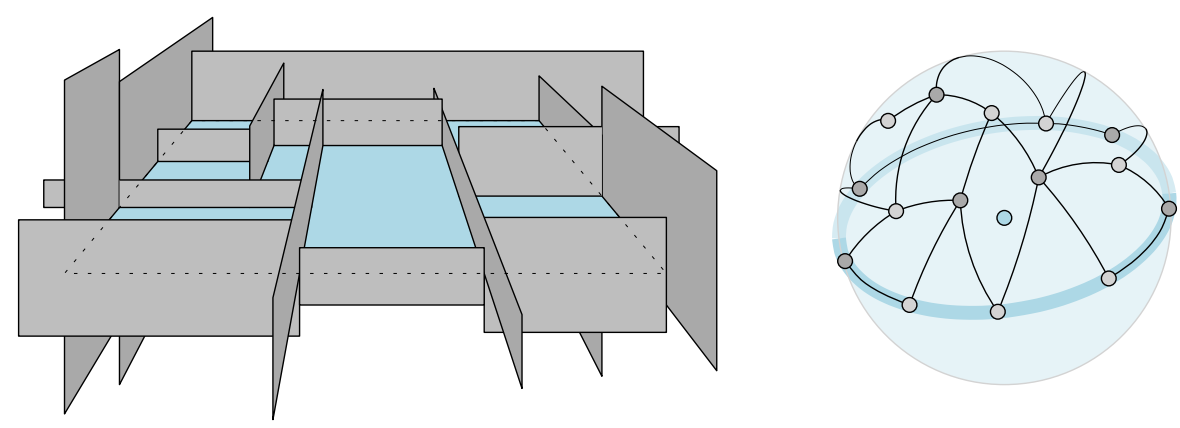

Fig. 3. Left: A $z$-rectangle (depicted in blue) in a Plattenbau with its touching rectangles intersecting its upper halfspace $H^{+}$. Right: The resulting crossing-free embedding on the upper hemisphere.

Item 3 A Plattenbau $\mathcal{R}$ can be transformed into a set $\mathcal{B}$ of boxes such that the touching graph of $\mathcal{R}$ is the intersection graph of $\mathcal{B}$ as follows: First, shrink each rectangle orthogonal to the $i$-axis by a small enough $\varepsilon>0$ in both dimensions different from $i$. As a result, we obtain a set of pairwise disjoint rectangles. Then, expand each such rectangle by $\varepsilon$ in dimension $i$. The obtained set $\mathcal{B}$ of boxes are again interiorly disjoint and all intersections are touchings.

Note that for Items 1 and 2 of Observation 1 it is crucial that $G$ is the touching graph and not the intersection graph. Moreover, Observation 1 allows to reject some graphs as Plattenbau graphs:

- $K_{4}$ is not a Plattenbau graph (by Item 1 of Observation 1),

- $K_{1,3,3}$ is not a Plattenbau graph (by Item 2 of Observation 1).

- The full subdivision of $K_{2^{2^{5}}+1}$ is not a Plattenbau graph (by Item 3 of Observation 1 and $[3]$ ).

In particular, some bipartite graphs are not Plattenbau graphs. Together with $K_{m, n}$ being a Plattenbau graph, this shows that the class of Plattenbau graphs is not closed under taking subgraphs; an unusual situation for touching graphs, which prevents us from solely focusing on edge-maximal Plattenbau graphs. To overcome this issue, we say that a Plattenbau $\mathcal{R}$ is proper if for any two touching rectangles an edge of one is contained in the other rectangle. (Note the ambiguity of the term "edge" here, which refers to a maximal line segment in the boundary of a rectangle as well as to a pair of adjacent vertices in a 
graph.) If $\mathcal{R}$ is proper, then each edge of the touching graph $G_{\mathcal{R}}$ can be removed by shortening one of the participating rectangles slightly. That is, the class of graphs with proper Plattenbau representations is closed under subgraphs.

We furthermore say that a Plattenbau $\mathcal{R}$ is boxed if six outer rectangles constitute the sides of a box that contains all other rectangles and all regions inside this box are also boxes. (A box is an axis-aligned full-dimensional cuboid, i.e., the Cartesian product of three bounded intervals of non-zero length.) For boxed Plattenbauten we use the additional convention that the edge-to-edge intersections of outer rectangles yield edges in the touching graph, even though these intersections contain no interior points. In particular, the outer rectangles of a proper boxed Plattenbau induce an octahedron in the touching graph.

Observation 2 The touching graph $G_{\mathcal{R}}$ of a proper Plattenbau $\mathcal{R}$ with $n \geq 6$ vertices has at most $4 n-12$ edges. Equality holds if and only if $\mathcal{R}$ is boxed.

Proof. For a proper Plattenbau $\mathcal{R}$ with touching graph $G_{\mathcal{R}}$ there is an injection from the edges of $G_{\mathcal{R}}$ to the edges of rectangles in $\mathcal{R}$ : For each edge $u v$ in $G_{\mathcal{R}}$ with corresponding rectangles $R_{u}, R_{v} \in \mathcal{R}$, take the edge of $R_{u}$ or $R_{v}$ that forms their intersection $R_{u} \cap R_{v}$. This way, each of the four edges of each of the $n$ rectangles in $\mathcal{R}$ corresponds to at most one edge in $G_{\mathcal{R}}$.

Moreover, if $\mathcal{R}$ contains at least two rectangles of each orientation, the bounding box of $\mathcal{R}$ contains at least 12 edges of rectangles in its boundary, none of which corresponds to an edge in $G_{\mathcal{R}}$. Thus, in this case $G_{\mathcal{R}}$ has at most $4 n-12$ edges. Otherwise, for one of the three orientations, $\mathcal{R}$ contains at most one rectangle in that orientation. In this case, $G_{\mathcal{R}}$ is a planar bipartite graph plus possibly one additional vertex. In particular, $G_{\mathcal{R}}$ has at most $2(n-1)-4+(n-1)<4 n-12$ edges, as long as $n \geq 6$.

Finally, in order to have exactly $4 n-12$ edges, the above analysis must be tight. This implies that $\mathcal{R}$ has at least two rectangles of each orientation and its bounding box contains exactly 12 edges of rectangles, i.e., $\mathcal{R}$ is boxed.

An immediate consequence of Observation 2 is that $K_{5,6}$ is a Plattenbau graph which has no proper Plattenbau representation. Contrary to the case of axis-aligned segments in $\mathbb{R}^{2}$, not every proper Plattenbau in $\mathbb{R}^{3}$ can be completed to a boxed Plattenbau; see Fig. 4 for a problematic example. This example can also easily be extended to give a graph $G$ that is the touching graph of a proper Plattenbau, but that is not a subgraph of any Plattenbau graph with a proper and boxed Plattenbau representation.

\section{Planar 3-Colorable Graphs}

Let us recall what shall be the main result of this section:

Theorem 1. Every 3-colorable planar graph is the touching graph of a proper Plattenbau. 


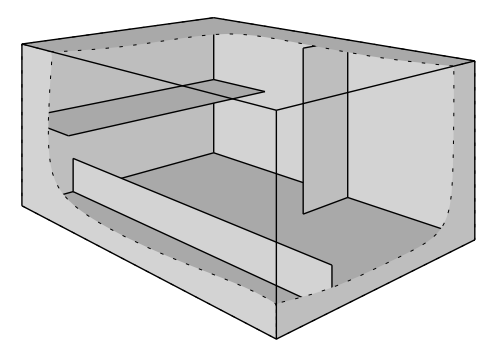

Fig. 4. A proper Plattenbau that cannot be augmented to a boxed Plattenbau.

The proof of this theorem is in several steps. First we introduce orthogonal surfaces and show that the dual graph of the skeleton of an orthogonal surface is a Plattenbau graph (Proposition 1). In the second step we characterize triangulations whose dual is the skeleton of an orthogonal surface (Proposition 2). One consequence of this is a natural very well-behaved partial order, namely a distributive lattice, on the set of orthogonal surfaces with given skeleton Corollary 1). We then show that a Plattenbau representation of a 3-colorable triangulation can be obtained by patching orthogonal surfaces in corners of orthogonal surfaces (Section 3.2).

We begin with an easy observation.

Observation 3 Every 3-colorable planar graph $G$ is an induced subgraph of a 3-colorable planar triangulation.

Proof (Sketch). Consider $G$ with a plane embedding. It is easy to find a 2connected 3-colorable $G^{\prime}$ which has $G$ as an induced subgraph.

Fix a 3-coloring of $G^{\prime}$. Let $f$ be a face of $G^{\prime}$ of size at least four and $c$ be a color such that at least three vertices of $f$ are not colored $c$. Stack a vertex $v$ inside $f$ and connect it to the vertices on $f$ that are not colored $c$. The new vertex $v$ is colored $c$ and the sizes of the new faces within $f$ are 3 or 4 . After stacking in a 4 -face, the face is either triangulated or there is a color which is not used on the newly created 4-face. A second stack triangulates it.

A plane triangulation $T$ is 3-colorable if and only if it is Eulerian. Hence, the dual graph $T^{*}$ of $T$ apart from being 3-connected, cubic, and planar is also bipartite. The idea of the proof is to find an orthogonal surface $\mathfrak{S}$ such that $T^{*}$ is the skeleton of $\mathfrak{S}$. This is not always possible but with a technique of patching one orthogonal surface in an appropriate corner of a Plattenbau representation obtained from another orthogonal surface, we shall get to a proof of the theorem.

Consider $\mathbb{R}^{3}$ with the dominance order, i.e., $x \leq y$ if and only if $x_{i} \leq y_{i}$ for $i=$ $1,2,3$. The join and meet of this distributive lattice are the componentwise max and min. Let $\mathcal{V} \subseteq \mathbb{R}^{3}$ be a finite antichain, i.e., a set of mutually incomparable points. The filter of $\mathcal{V}$ is the set $\mathcal{V}^{\uparrow}:=\left\{x \in \mathbb{R}^{3} \mid \exists v \in \mathcal{V}: v \leq x\right\}$ and the boundary $\mathfrak{S}_{\mathcal{V}}$ of $\mathcal{V}^{\uparrow}$ is the orthogonal surface generated by $\mathcal{V}$. The left part of Fig. 5 shows an example in $\mathbb{R}^{3}$. The nine vertices of the generating set $\mathcal{V}$ are emphasized. 

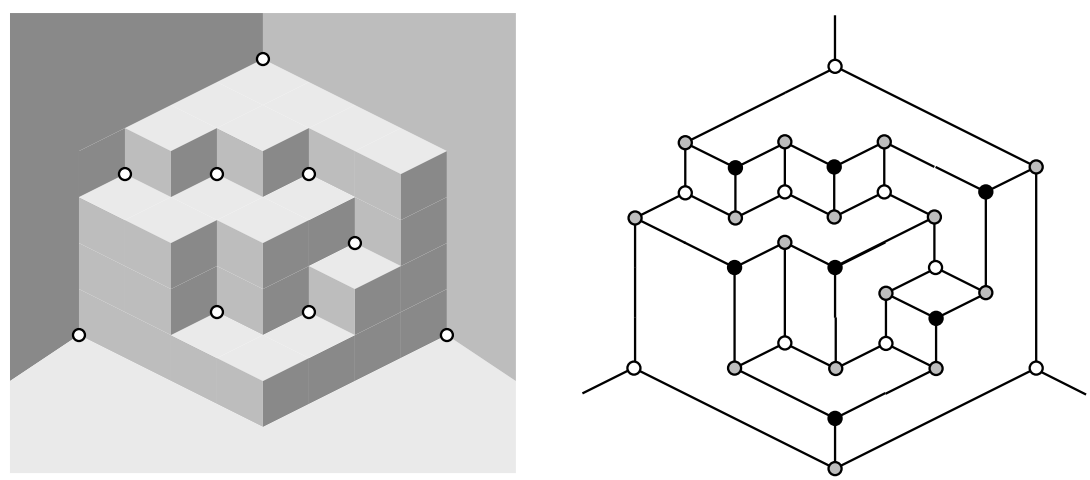

Fig. 5. An orthogonal surface and its skeleton (vertex $v_{\infty}$ omitted).

Orthogonal surfaces have been studied by Scarf [15] in the context of test sets for integer programs. They later became of interest in commutative algebra, cf. the monograph of Miller and Sturmfels 21. Miller 20 observed the connections between orthogonal surfaces, Schnyder woods and the Brightwell-Trotter Theorem about the order dimension of polytopes, see also 8.

A maximal set of points of an orthogonal surface which is constant in one of the coordinates is called a flat. A non-empty intersection of two flats is an edge. A point contained in three flats is called a vertex. An edge incident to only one vertex is a ray. We will only consider orthogonal surfaces obeying the following non-degeneracy conditions: (1) The boundary of every bounded flat is a simple closed curve. (2) There are exactly three rays. Note that from (1) it can be deduced that every vertex is contained in exactly three flats.

The skeleton graph $G_{\mathfrak{S}}$ of an orthogonal surface consists of the vertices and edges of the surface, in addition there is a vertex $v_{\infty}$ which serves as second vertex of each ray. The skeleton graph is planar, cubic, and bipartite. The bipartition consists of the maxima and minima of the surface in one class and of the saddle vertices in the other class. The vertex $v_{\infty}$ is a saddle vertex. The dual of $G_{\mathfrak{S}}$ is a triangulation with a designated outer face, the dual of $v_{\infty}$.

The generic structure of a bounded flat is as shown in Fig. 6, the boundary consists of two zig-zag paths sharing the two extreme points of the flat. The minima of the lower zig-zag are elements of the generating set $\mathcal{V}$, they are minimal elements of the orthogonal surface $\mathfrak{S}$. The maxima of the upper zig-zag are maximal elements of $\mathfrak{S}$, they can be considered to be dual generators.

With the following proposition we establish a first connection between orthogonal surfaces and Plattenbau graphs.

Proposition 1. The dual triangulation of the skeleton of an orthogonal surface $\mathfrak{S}$ is a Plattenbau graph and admits a proper Plattenbau representation.

Proof. Choose a point not on $\mathcal{V}$ on each of the three rays of $\mathfrak{S}$ and call these points the extreme points of their incident unbounded flats. 


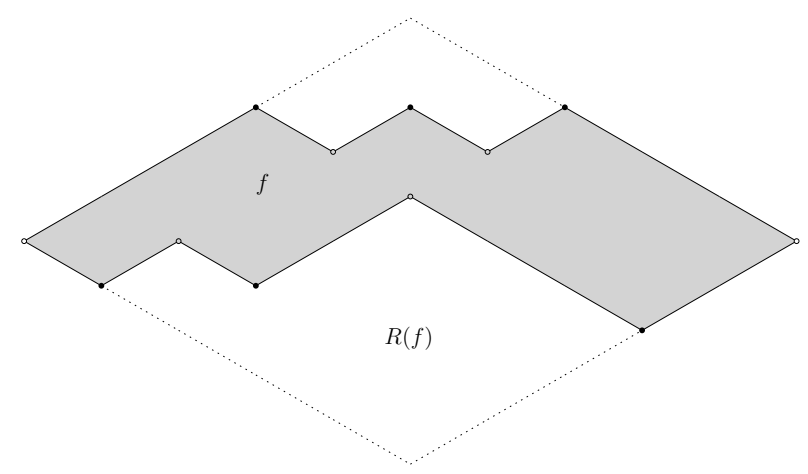

Fig. 6. Generic flat $f$ and the spanned rectangle $R(f)$.

The two extreme points $a_{f}, b_{f}$ of a flat $f$ of $\mathfrak{S}$ span a rectangle $R(f)$. Note that the other two corners of $R(f)$ are $\max \left(a_{f}, b_{f}\right)$ and $\min \left(a_{f}, b_{f}\right)$. We claim that the collection of rectangles $R(f)$ is a weak rectangle contact representation of the dual triangulation $T$ of the skeleton of $\mathfrak{S}$. Here weak means that the contacts of pairs of rectangles of different orientation can be an edge to edge contact. If $f$ and $f^{\prime}$ share an edge $e$ of the skeleton, then since one of the ends of $e$ is a saddle point of $\mathfrak{S}$ and thus extreme in two of its incident flats, it is extreme for at least one of $f$ and $f^{\prime}$. This shows that $e$ is contained in the boundary of at least one of the rectangles $R(f), R\left(f^{\prime}\right)$, i.e., the intersection of the open interiors of the rectangles is empty.

Let $f$ and $f^{\prime}$ be two flats which share no edge. Let $H_{f}$ and $H_{f^{\prime}}$ be the supporting planes. If $f$ is contained in an open halfspace $O$ defined by $H_{f^{\prime}}$, then $\max \left(a_{f}, b_{f}\right)$ and $\min \left(a_{f}, b_{f}\right)$, the other two corners of $R(f)$, are also in $O$, hence $R(f) \subset O$ and $R(f) \cap R\left(f^{\prime}\right)=\emptyset$. If $f$ intersects $H_{f^{\prime}}$ and $f^{\prime}$ intersects $H_{f}$, then consider the line $\ell=H_{f^{\prime}} \cap H_{f}$. This line is parallel to one of the axes, hence it intersects $\mathfrak{S}$ in a closed interval $I_{\mathfrak{S}}$. If $I_{f}$ and $I_{f^{\prime}}$ are the intervals obtained by intersecting $\ell$ with $f$ and $f^{\prime}$ respectively, then one of them equals $I_{\mathfrak{S}}$ and the other is an edge of the skeleton of $\mathfrak{S}$, i.e., $\left(f, f^{\prime}\right)$ is an edge of $T$.

It remains to expand some of the rectangles to change weak contacts into true contacts. Let $e=f \cap f^{\prime}$ be an edge such that the contact of $R(f)$ and $R\left(f^{\prime}\right)$ is weak. Select one of $f$ and $f^{\prime}$, say $f$. Now expand the rectangle $R(f)$ with a small parallel shift of the boundary segment containing $e$. This makes the contact of $R(f)$ and $R\left(f^{\prime}\right)$ a true contact. The expansion can be taken small enough as to avoid that new contacts or intersections are introduced. Iterating over the edges we eventually get rid of all weak contacts. See Fig. 7 for an illustration.

Recall that we aim at realizing $T^{*}$, the dual of the 3 -colorable triangulation $T$ as the skeleton of an orthogonal surface. Since $T$ is Eulerian its dual $T^{*}$ is bipartite. Let $U$ (black) and $U^{\prime}$ (white) be the bipartition of the vertices of $T^{*}$ such that the dual $v_{\infty}$ of the outer face of $T$ is in $U$. The critical task is to assign two extreme vertices to each face of $T^{*}$ which does not contain $v_{\infty}$. This has to 


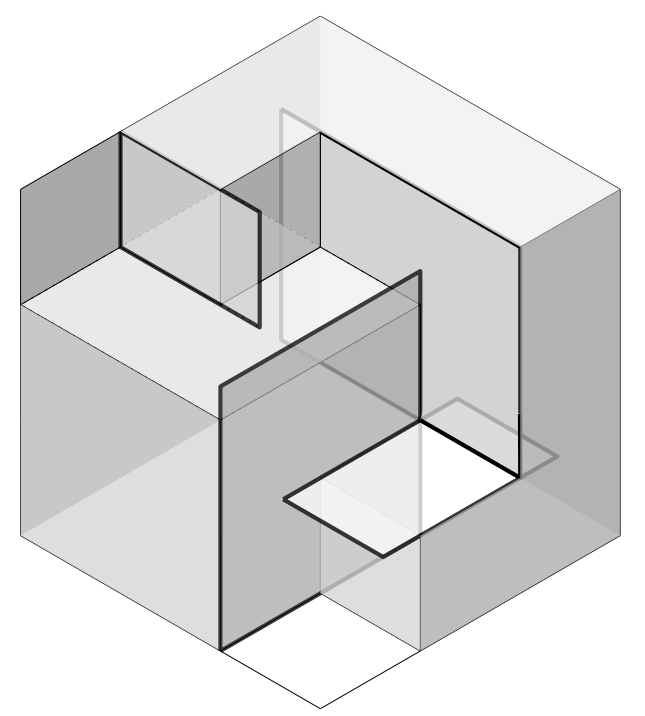

Fig. 7. Replacing flats by rectangles and expanding in order to avoid weak contacts.

be done so that each vertex in $U$ (except $v_{\infty}$ ) is extremal for exactly two of the faces.

To solve the assignment problem we will work with an auxiliary graph $H_{T}$. The faces of $T^{*}$ which do not contain $v_{\infty}$ are the interior vertices of $T$, we denote this set with $V^{\circ}$. As the vertices of $T^{*}$ are the facial triangles of $T$, we think of $U$ as representing the black triangles of $T$. We also let $U^{\circ}=U-v_{\infty}$, this is the set of bounded black triangles of $T$. The vertices of $H_{T}$ are $V^{\circ} \cup U^{\circ}$ the edges of $H_{T}$ correspond to the incidence relation in $T^{*}$ and $T$ respectively, i.e., $v, u$ with $v \in V^{\circ}$ and $u \in U^{\circ}$ is an edge if vertex $v$ is a corner of the black triangle $u$. A valid assignment of extreme vertices is equivalent to an orientation of $H_{T}$ such that each vertex $v \in V^{\circ}$ has outdegree two and each vertex $u \in U^{\circ}$ has indegree two, i.e., the outdegrees of the vertices are prescribed by the function $\alpha$ with $\alpha(v)=2$ for $v \in V^{\circ}$ and $\alpha(u)=\operatorname{deg}(u)-2$ for $u \in U^{\circ}$. Since $\left|V^{\circ}\right|=\left|U^{\circ}\right|=n-3$ it is readily seen that the sum of the $\alpha$-values of all vertices equals the number of edges of $H_{T}$.

Orientations of graphs with prescribed out-degrees have been studied e.g. in 9 , there it is shown that the following necessary condition is also sufficient for the existence of an $\alpha$-orientation. For all $W \subset V^{\circ}$ and $S \subset U^{\circ}$ and $X=W \cup S$

$$
\sum_{x \in X} \alpha(x) \leq|E[X]|+|E[X, \bar{X}]|
$$

Here $E[X]$ and $E[X, \bar{X}]$ denote the set of edges induced by $X$, and the set of edges in the cut defined by $X$, respectively.

Inequality $\alpha$ does not hold for all triangulations $T$ and all $X$. We next identify specific sets $X$ violating the inequality, they are associated to certain 
badly behaving triangles, we call them babets. In Proposition 2 we then show that babets are the only obstructions for the validity of $(\alpha)$.

Let $\Delta$ be a separating triangle of $T$ such that the faces of $T$ bounding $\Delta$ from the outside are white. Let $W$ be the set of vertices inside $\Delta$ and let $S$ be the collection of black triangles of $T$ which have all vertices in $W$. We claim that $X=$ $W \cup S$ is violating $(\alpha)$. If $|W|=k$ and $|S|=s$, then $\sum_{x \in X} \alpha(x)=2|W|+|S|=$ $2 k+s$. The triangulation whose outer boundary is $\Delta$ has $2(k+3)-4$ triangles, half of them, i.e., $k+1$, are black and interior. The right side of $(\alpha)$ is counting the number of incidences between $W$ and black triangles. Black triangles in $\Delta$ have $3(k+1)$ incidences in total. There are $k+1-s$ black triangles have an incidence with a corner of $\Delta$ and 3 of them have incidences with two corners of $\Delta$. Hence the value on the right side is $3(k+1)-(k+1-s)-3=2 k+s-1$. This shows that the inequality is violated. A separating triangle $\Delta$ of $T$ with white touching triangles on the outside is a babet.

Proposition 2. If $T$ has no babet, then there is an orientation of $H_{T}$ whose outdegrees are as prescribed by $\alpha$.

Before we prove Proposition 2 and Theorem 1, let us briefly summarize the procedure.

First, we construct a Plattenbau representation in the babet-free case $\mathrm{Sec}-$ tion 3.1 based on an auxiliary graph $G$ arising from the bipartition of $T^{*}$, and a Schnyder wood $S$ for $G$. We then find an orthogonal surface $\mathfrak{S}$ based on the Schnyder wood $S$ and show that the skeleton $G_{\mathfrak{S}}$ of $\mathfrak{S}$ is $T^{*}$, which together with Proposition 1 gives a Plattenbau for $T$. Then (Section 3.2), in case $T$ contains some babets, we cut the triangulation $T$ along an innermost babet, find orthogonal surfaces for the orthogonal surfaces for the inside and outside, and patch the former into a saddle point of the latter.

Now, let us start with the proof of Proposition 2

Proof. Suppose that there is an $X=W \cup S$ violating inequality $\alpha$. We are going to modify $X$ in several steps always maintaining the property that the inequality is violated. At the end we will be able to point to a babet in $T$.

Suppose there is a $u \in S$ with $a \leq 2$ neighbors in $W$. Let $X^{\prime}=X-u$ when going from $X$ to $X^{\prime}$ the left side of $\sqrt{\alpha}$ is loosing $\operatorname{deg}(u)-2$ while on the right side we loose the $\operatorname{deg}(u)-a$ edges of $H_{T}$ which are incident to $u$ but not to $W$. Since $a \leq 2$ the set $X^{\prime}$ is violating. From now on we assume that every $u \in S$ has 3 neighbors in $W$, in particular $\alpha(u)=1$.

Now the left side of $(\alpha)$ equals $2|W|+|S|$ and for the right side we have $|E[X]|=$ $3|S|$ and $E[X, \bar{X}]$ contains no edge incident to $S$. We define $\partial W=|E[X, \bar{X}]|$ the notation indicates that we only have to care of boundary edges of $W$. The assumption that Inequality $(\alpha)$ is violated then becomes $2|W|+|S|>3|S|+\partial W$ or equivalently

$$
2|W|>2|S|+\partial W .
$$

We can assume that the subgraph of $H_{T}$ induced by $X$ is connected, otherwise a connected component would also violate. Let $|W|=k$ and $|S|=s$. The set $S$ is 
a set of black triangles in the triangulation $T$ and $W$ is the set of vertices of these triangles. Let $T_{S}$ be the plane embedding of all edges of triangles of $S$ as seen in $T$. Classify the faces of $T_{S}$ as black triangles, white triangles and big faces, and let their numbers be $s, t$ and $g$, respectively. We consider the outer face of $T_{S}$ a big face independent of its size, therefore, $g \geq 1$. Consider the triangulation $T_{S}^{+}$ obtained by stacking a new vertex in each big face and connecting it to all the angles of the face, i.e., the degree of the vertex $z_{f}$ stacked into face $f$ equals the length $r_{f}$ of the boundary of $f$. Note that $T_{S}^{+}$may have multi-edges but every face of $T_{S}^{+}$is a triangle so that Euler's formula holds. Let $R=\sum_{f} r_{f}$ be the sum of degrees of the stack vertices. Since $T_{S}^{+}$has $k+g$ vertices it has $2(k+g)-4$ faces. However, we also know that $T_{S}^{+}$has $s+t+R$ faces. Counting the edges incident to the triangles of $S$ we obtain $3 s=3 t+R$. Using this to eliminate $t$ we obtain $2(k+g)-4=2 s+\frac{2 R}{3}$, i.e., $2|W|=2|S|+4-2 g+\frac{2 R}{3}$. With (D) this implies $4+\frac{2 R}{3}>\partial W+2 g$.

Claim 1. $R$ is divisible by 3 .

Proof of Claim. Actually we prove that each $r_{f}$ is divisible by 3 . Let $Y$ be a collection of triangles in a 3-colorable triangulation and let $\gamma$ be the boundary of $Y$, i.e., $\gamma$ is the set of edges incident to exactly one triangle from $Y$. Let $Y_{b}$ and $Y_{w}$ be the black and white triangles in $Y$ and let $\gamma_{b}$ and $\gamma_{w}$ be the edges of $\gamma$ which are incident to black and white triangles of $Y$. Double counting the number of edges in $Y$ we get $3\left|Y_{b}\right|+\left|\gamma_{w}\right|=3\left|Y_{w}\right|+\left|\gamma_{s}\right|$, hence, $\left|\gamma_{w}\right| \equiv\left|\gamma_{s}\right|$ mod 3. In our case $\left|\gamma_{s}\right|=0$ and depending on the chosen $Y$ either $R=\left|\gamma_{w}\right|$ or $r_{f}=\left|\gamma_{w}\right|$.

Let $\gamma$ be the boundary cycle of a big face of $T_{S}$ which is not the outer face. We have seen that $|\gamma| \equiv 0 \bmod 3$. We are interested in the contribution of $\gamma$ to $\partial W$, i.e., in the number of incidences of vertices of $\gamma$ with black triangles in the inside. For convenience we can use multiple copies of a vertex to make $\gamma$ simple. The claim is that the contribution of $\partial \gamma$ to $\partial W$ is at least $2|\gamma| / 3$. If this is shown we can add all the inner black triangles of $\gamma$ to $S$ and all the inner vertices to $W$, the left side of the violator inequality $4+\frac{2 R}{3}>\partial W+2 g$ is thereby reduced by $2|\gamma| / 3$ and the right side is reduced by at least $2|\gamma| / 3+2$, i.e., violators are preserved.

To prove the claim consider the interior triangulation $T_{\gamma}$ of the simple cycle $\gamma$. Suppose $\partial \gamma \leq 2|\gamma| / 3$. Then on $\gamma$ we find an ear, this is a vertex which has no incidence to a black triangle of $T_{\gamma}$, i.e., its degree in $T_{\gamma}$ is 2 . If $b$ is an ear and $a$, $c$ are the neighbors of $b$ on $\gamma$, then $a, c$ is an edge and there is a black triangle $a c x$ in $T_{\gamma}$. An ear is reducible if $b$ has a neighbor on $\gamma$ which is only incident to a single black triangle in $T_{\gamma}$. Assume that $c$ is such a neighbor of $b$, then the second neighbor $d$ of $c$ on $\gamma$ has an edge to $x$. If $x$ is the unique common neighbor of $a$ and $d$, then we delete $b$ and $c$ and identify the edges $x a$ and $x d$. This results in a cycle $\gamma^{\prime}$ with $\left|\gamma^{\prime}\right|=|\gamma|-3$. We call this an ear reduction with center $x$. Fig. 8 shows sketches of reducible ears. Note that in one case $\partial \gamma \geq \partial \gamma^{\prime}+2$ (depending on whether $x$ belongs to $\gamma$ or not) and in the other $\partial \gamma=\partial \gamma^{\prime}+3$. 

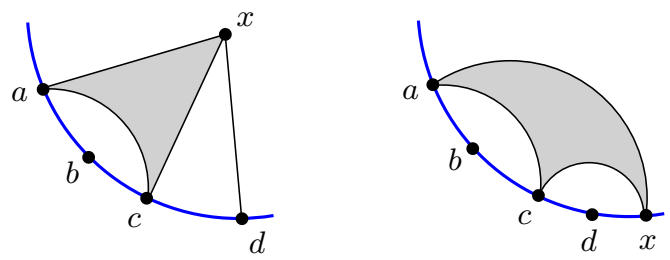

Fig. 8. Two sketches of reducible ears. The right one is the special case where $d$ is an ear.

If $b$ is a reducible edge but $a$ and $d$ share several neighbors, then there is an extreme common neighbor $y$ with the property that the cycle $a, b, c, d, y$ encloses all the common neighbors of $a$ and $d$. In this case we perform an ear reduction with center $y$, indeed, due to the mod 3 parity exactly one of the interior triangles of ay and $y d$ is black. Again we get a a cycle $\gamma^{\prime}$ with $\left|\gamma^{\prime}\right|=|\gamma|-3$ and $\partial \gamma \geq \partial \gamma^{\prime}+2$.

After a series of reductions we obtain a cycle $\gamma^{\prime}$ which has no reducible ear. Let each vertex with at least two incidences with black triangles discharge $\frac{1}{2}$ to each neighbor, then all vertices have a weight of at least 1 . Hence $\partial \gamma^{\prime} \geq\left|\gamma^{\prime}\right|$ and $\partial \gamma \geq \partial \gamma^{\prime}+2 m \geq\left|\gamma^{\prime}\right|+2 m=|\gamma|-3 m+2 m \geq 2|\gamma| / 3$. This completes the proof of the claim.

We have already seen that the claim implies that we may assume that $g=1$, i.e., the violator $T_{S}$ only has a single big face, the outer face $f_{\infty}$. We write $r_{f_{\infty}}=3 \rho$, the condition for violation becomes $\partial W<2 \rho+2$.

Let $\gamma$ be the boundary cycle of the unique big face. While the outer face of $T$ has to be contained in the big face we prefer to think of a drawing of $T_{S}$ such that the big face is the interior of $\gamma$. It will be crucial, however, that in the inner triangulation of $\gamma$ inherited from $T$ there is a special black triangle $\delta_{\infty}$. The goal is to find a babet in the interior of $\gamma$, we use induction on $\rho$.

In the case $\rho=1$ the triangle $\gamma$ has $\partial W<4$ incidences with black triangles from the inside. The unique configuration with this property is shown on the left in Fig. 9. Since the black triangle $\delta_{\infty}$ is not yet in the picture one of the triangles must be separating. If the separating triangle were not the central one it would lead to an increase of $\partial W$. Hence the white central triangle is separating, i.e., a babet.

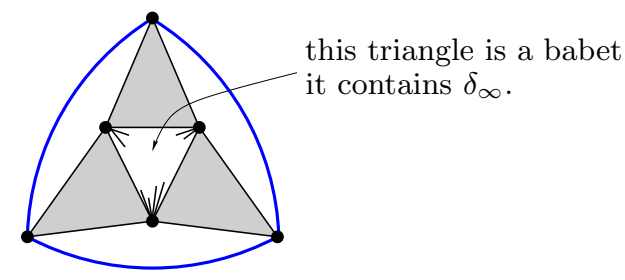

Fig. 9. Illustration for the case $\rho=1$. 
Now let $\rho \geq 2$. Assuming a black incident triangle for every vertex of $\gamma$ we obtain $\partial W \geq 3 \rho \geq 2 \rho+2$. Therefore, on $\gamma$ we find an ear. As above we aim for an ear reduction. Let $b$ be a reducible ear with neighbors $a$ and $c$ such that $c$ is only incident to a single black triangle $a c x$ inside $\gamma$ and $d$ is the second common neighbor of $c$ and $x$. If $x$ is the unique common neighbor of $a$ and $d$, then the reduction leads to a decrease of $\partial W$ by 2 or 3 and a decrease of $\rho$ by 1 , whence the reduced cycle remains violating.

If $b$ is reducible but $a$ and $d$ share several neighbors, then there we aim at a reduction whose center $y$ is the extreme common neighbor of $a$ and $d$. If this cycle does not enclose the black triangle $\delta_{\infty}$ we can apply the reduction with center $y$.

The described reductions may decrease $\rho$ until it is 1 whence there is a babet. In fact we will complete the proof by showing that when no reduction is possible and $\rho>1$, then the violator inequality is not fulfilled.

We first discuss the case where $b$ is reducible but $a$ and $d$ share several neighbors and $\delta_{\infty}$ is enclosed in the cycle $a, b, c, d, y$ where $y$ is the extreme common neighbor of $a$ and $d$. We show that in this case we can find a cycle $\gamma^{\prime}$ of length 6 which is a violator, i.e., $\partial \gamma<6$.
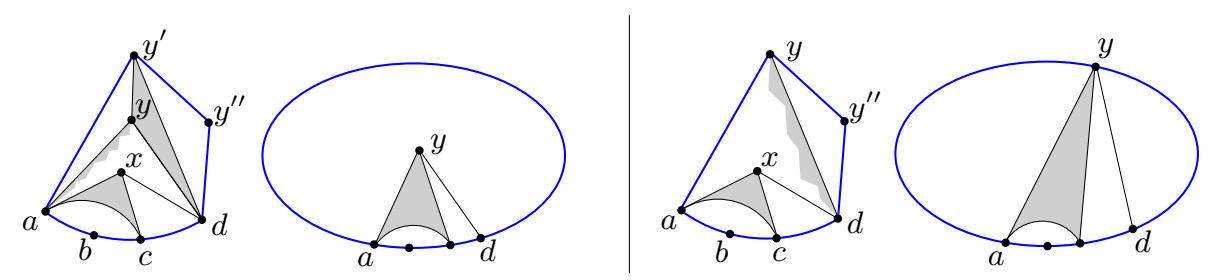

Fig. 10. Illustration for the subconfigurations generated from a cycle $a, b, c, d, y$ enclosing $\delta_{\infty}$.

Suppose $y$ is an interior of $\gamma$ in this case we take the cycle $a, b, c, d, y$ add a new common neighbor $y^{\prime}$ for $a, d, y$ and an ear $y^{\prime \prime}$ over the black triangle containing $y^{\prime}$. This yields a 6 cycle $\gamma^{\prime \prime}$ as shown in Fig. 10 (left 1). In the interior of $\gamma$ we replace the triangles inside $a, b, c, d, y$ by the 3 triangles of a reducible ear, see Fig. 10 (left 2) and refer to the cycle with the simplified interior as $\gamma^{\prime}$. Now we compare $\partial \gamma$ with $\partial \gamma^{\prime}$ and $\partial \gamma^{\prime \prime}$ and use the bound previously shown in the claim for $\gamma^{\prime}$, i.e., $\partial \gamma^{\prime} \geq 2\left|\gamma^{\prime}\right| / 3=2|\gamma| / 3$. Taking into account that on each of $\gamma^{\prime}$ and $\gamma^{\prime \prime}$ we see two incidences with black triangles which are not counted in $\partial \gamma$ we get $2|\gamma| / 3+2>\partial \gamma \geq\left(\partial \gamma^{\prime \prime}-2\right)+\left(\partial \gamma^{\prime}-2\right) \geq \partial \gamma^{\prime \prime}+2|\gamma| / 3-4$. Hence $\partial \gamma^{\prime \prime}<6$, whence $\gamma^{\prime \prime}$ is also a violator.

If $y$ is a vertex on the cycle $\gamma$ we add a new ear $y^{\prime}$ either over ay or over $d y$ depending on which is incident to a black triangle. This yields a 6 -cycle $\gamma^{\prime \prime}$ as shown in Fig. 10 (right 1). In the interior of $\gamma$ we replace the triangles inside $a, b, c, d, y$ by the 3 triangles of a reducible ear, see Fig. 10 (right 2) and refer to the cycle with the simplified interior as $\gamma^{\prime}$. Taking into account that on $\gamma^{\prime}$ and 
we see three incidences with black triangles which are not counted in $\partial \gamma$ we get $2|\gamma| / 3+2>\partial \gamma \geq \partial \gamma^{\prime \prime}+\left(\partial \gamma^{\prime}-3\right) \geq \partial \gamma^{\prime \prime}+2|\gamma| / 3-3$. Hence $\partial \gamma^{\prime \prime}<5$, whence $\gamma^{\prime \prime}$ is also a violator.

If $\rho \geq 3$ and there are no reducible ears, then both neighbors of each ear vertex have two incidences with black triangles. Let each vertex with at least two incidences with black triangles discharge $\frac{1}{2}$ to the neighbors, then all vertices have a weight of at least 1 . We get $\partial W \geq 3 \rho>2 \rho+2$ and the example is not a violator.

For the case $\rho=2$ we consider circular sequences $\left(s_{1}, s_{2}, s_{3}, s_{4}, s_{5}, s_{6}\right)$ with $\sum_{i} s_{i}<2 \rho+2=6$ such that there is an inner Eulerian triangulation of a 6 -gon with only white triangles touching the 6 -gon and $s_{i}$ black incident triangles at vertex $v_{i}$ of the 6 -gon. A vertex $v_{i}$ with $s_{i}=0$ is an ear. Clearly, the circular sequence has no 00 subsequence. With two ears which share a neighbor of degree 1, i.e., with a 010 subsequence, we have the graph $T_{1}$ from Fig. 11 this triangulation does not occur in our setting since it does not contain a black $\delta_{\infty}$ which is vertex disjoint from the outer 6-gon. Making any of the four triangles of $T_{1}$ separating so that it can accommodate $\delta_{\infty}$ in its interior would make $\sum_{i} s_{i} \geq 6$.
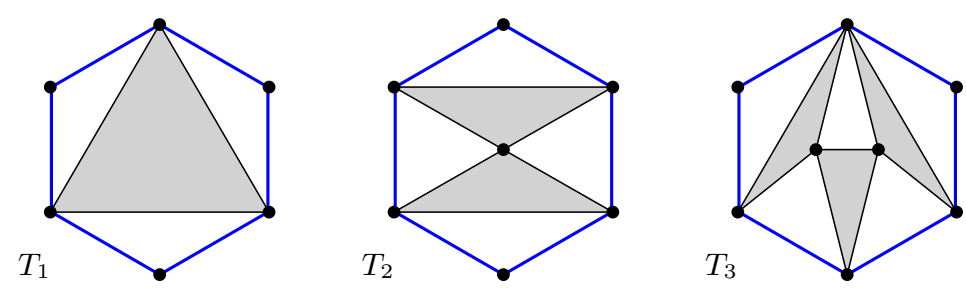

Fig. 11. Three triangulations with $\rho=2$ and $\partial W<6$.

With two ears which are not adjacent we have $T_{2}$. Again there is no $\delta_{\infty}$ in $T_{2}$ and making a triangle separating would make $\sum_{i} s_{i} \geq 6$. It remains to look at sequences without 00 , and 010 , and 101 but $\sum_{i} s_{i} \leq 5$. The sequence 201110 is the unique sequence with these properties and there is a unique corresponding triangulation $T_{3}$. As with $T_{1}$ and $T_{2}$ there is no $\delta_{\infty}$ in $T_{3}$ and making a triangle separating would make $\sum_{i} s_{i} \geq 6$.

\subsection{Plattenbau Representations in the Babet-Free Case}

Let $T$ be a 3-colorable triangulation which has no babet. Due to Proposition 2 we find the $\alpha$-orientation of $H_{T}$. This orientation can be represented on the dual $T^{*}$ of $T$ as a collection $\mathcal{C}$ of cycles such that every face which is not incident to $v_{\infty}$ contains exactly one fragment of a cycle which leaves the face in distinct vertices of $U^{\circ}$ and every vertex of $U^{\circ}$ is covered by one of the cycles (Eppstein and Mumford [6] refer to this structure as cycle cover). The connected components of $\mathbb{R}^{2} \backslash \mathcal{C}$ can be two-colored in white and pink such that the two sides of each cycle have distinct colors, the two-coloring is unique if we want the unbounded 
region to be colored white. This two-coloring of the plane induces a partition of the vertices of $U^{\prime}$ into $U_{w}^{\prime}, U_{p}^{\prime}$ where $U_{w}^{\prime}$ consists of all vertices living in white regions and $U_{p}^{\prime}$ consists of the vertices in pink regions, see Fig. 12 for an example. An important property of the partition is that every vertex of $u \in U^{\circ}$ is adjacent to vertices from both classes $U_{w}^{\prime}$ and $U_{p}^{\prime}$. This follows form the fact that a cycle $C$ from $\mathcal{C}$ contains $u$, whence two of the edges of $u$ are on one side and the third is on the other side of $C$.

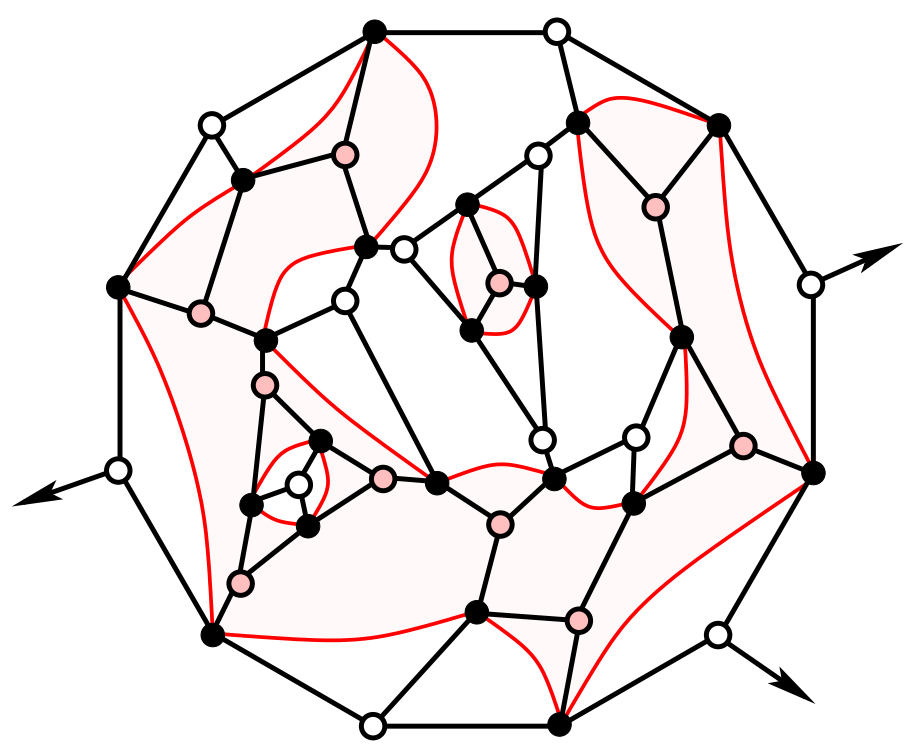

Fig. 12. Example of a $T^{*}$ with a cycle cover of $U^{\circ}$ and the induced partition of $U^{\prime}$.

The partition $U_{w}^{\prime}, U^{\circ}, U_{p}^{\prime}$ of the vertices of $T^{*} \backslash\left\{v_{\infty}\right\}$ corresponds to the partition into minima, saddle points, maxima of the vertices of the orthogonal surface with skeleton $T^{*}$. To construct this orthogonal surface we first define a 3-connected planar graph $G$ whose vertex set is $U_{w}^{\prime}$, the edges of $G$ are in bijection with $U^{\circ}$ and each bounded face of $G$ contains exactly one vertex of $U_{p}^{\prime}$. This graph $G$ will be decorated with a Schnyder wood, i.e., an orientation of the edges which obeys the following rules:

(W1) On the outer face of $G$ there are three special vertices $a_{r}, a_{g}, a_{b}$ colored red, green, and blue in clockwise order. For $c \in\{r, g, b\}$ vertex $a_{c}$ is equipped with an outward oriented half-edge of color $c$.

(W2) Every edge $e$ is oriented in one or in two opposite directions. The directions of edges are colored such that if $e$ is bioriented the two directions have distinct colors.

(W3) Every vertex $v$ has outdegree one in each color $c \in\{r, g, b\}$. The edges $e_{r}, e_{g}, e_{b}$ leaving $v$ in colors $r, g, b$ occur in clockwise order. Each edge 
entering $v$ in color $c$ enters $v$ in the sector bounded by the two $e_{i}$ with colors different from $c$ (see Fig. 13).

(W4) There is no interior face whose boundary is a directed cycle in one label.

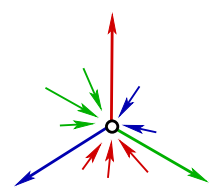

Fig. 13. Illustration for the vertex condition (W3)

The construction of $G$ and the Schnyder wood $S$ is in several steps. The first step is to define a coloring of the edges of $T^{*}$. Let $T$ be colored with $r, g, b$ such that on the outer face these colors appear clockwise in the given order. Note that this implies that all white triangles see $r, g, b$ in clockwise order and all black triangles see $r, g, b$ in counterclockwise order. The coloring of $T$ induces a 3-coloring of the edges: for edge $e=v, v^{\prime}$ use the unique color which is not used for $v$ and $v^{\prime}$. This edge coloring of $T$ can be copied to the dual edges. This yields an edge coloring of $T^{*}$ such that each vertex in $U^{\prime}$ is incident to edges of colors $r, g, b$ in clockwise order. Delete $v_{\infty}$ from $T^{*}$ but keep the edges incident to $v_{\infty}$ as half edges at their other endpoint, we denote the obtained graph as $T_{\infty}^{*}$. The neighbors of $v_{\infty}$ are the special vertices $a_{r}, a_{g}, a_{b}$ for the Schnyder wood.

Next we introduce some new edges. For every $u \in U^{\circ}$ which is adjacent to only one vertex $v$ of $U_{w}^{\prime}$ we identify the unique face $f$ which is adjacent to $u$ but not to $v$. Connect $u$ to a vertex $v^{\prime}$ on the boundary of $f$ which belongs to $U_{w}^{\prime}$. Note that the edge $u v^{\prime}$ is intersected by a cycle from $\mathcal{C}$ and that both faces obtained by cutting $f$ via $u, v^{\prime}$ contain the vertex $v^{\prime} \in U_{w}^{\prime}$. This shows that we can add edges to all vertices of $U^{\circ}$ which are adjacent to only one vertex in $U_{w}^{\prime}$ without introducing crossings. The color of $v u$ is copied to the new edge $u v^{\prime}$. Fig. 14 exemplifies the coloring of $T_{\infty}^{*}$ together with the additional edges.

Now remove all the edges incident to vertices in $U_{p}^{\prime}$. In the remaining graph all the vertices of $U^{\circ}$ are of degree 2 . We remove these 'subdivision' vertices and melt the two edges into one. The result is $G$. We claim that orienting the three edges of $v \in U_{p}^{\prime}$ which come from an edge of $T_{\infty}^{*}$ as outgoing we obtain a Schnyder wood of $G$. Indeed (W1), (W2) and (W3) follow directly or from what we have already said. For (W4) we need a little argument. Consider a monochromatic edge $v v^{\prime}$ and let $f$ be one of the faces of $G$ containing this edge on the boundary. The other edge on the boundary of $f$ which contains $v^{\prime}$ is either incoming in the same color or outgoing in a different color. This shows that there is no monochromatic directed facial cycle containing $v v^{\prime}$. Now consider a face $f$ of $G$ which has no monochromatic edge. Note that $f$ is a face of the graph obtained from $T_{\infty}^{*}$ by deleting all the edges incident to vertices in $U_{p}^{\prime}$. Let $w \in U_{p}^{\prime}$ be a vertex in the interior of $f$ such that in $T_{\infty}^{*}$ there is an edge $w u$ with $u \in U^{\circ}$ 


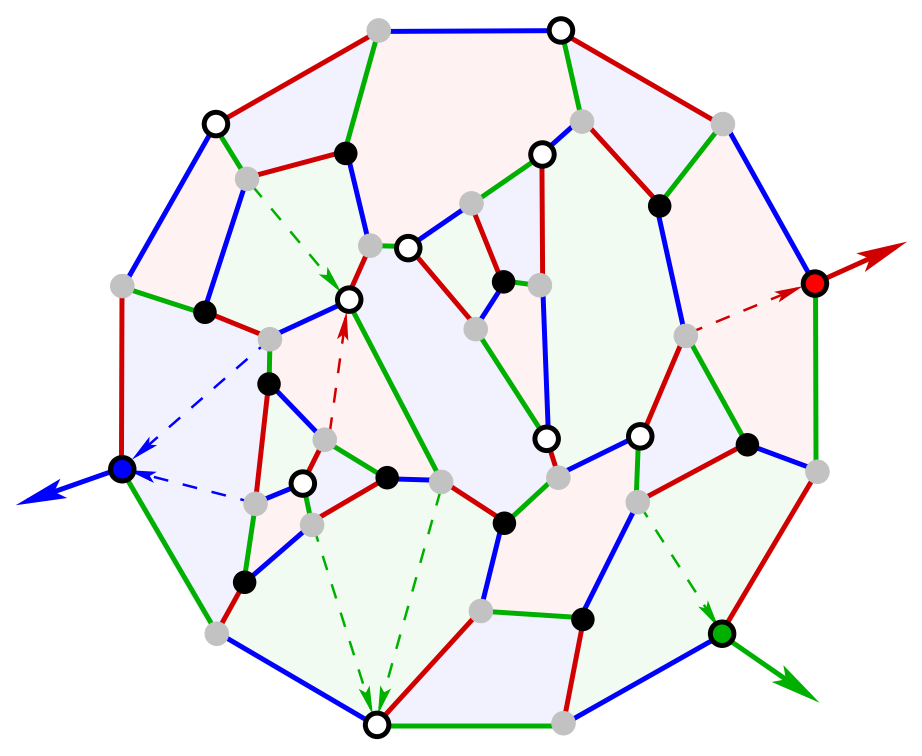

Fig. 14. The coloring of the edges of $T_{\infty}^{*}$ obtained from the 3-coloring of $T$. The dashed arrows are the additional edges.

being on the boundary $\partial f$ of $f$. Let $c \in\{r, g, b\}$ be the color of the edge $w u$. Vertex $u$ has two neighbors $v, v^{\prime}$ on $\partial f$. By looking at the colors of edges of $T_{\infty}^{*}$ we see that at $v$ and $v^{\prime}$ the incident edge on $\partial f$ which is different from $v v^{\prime}$ has color $c$. In the Schnyder wood we therefore see these outgoing in color $c$ at $v$ and $v^{\prime}$ one of them being oriented clockwise, the other counterclockwise on the boundary of $f$. This shows that $\partial f$ supports no monochromatic directed cycle.

For the following we rely on the theory of Schnyder woods for 3-connected planar graphs, see e.g. 7] or 12 or 9. In the Schnyder wood $S$ on $G$ for every vertex $v$ and every color $c \in\{r, g, b\}$ there is a directed path $P_{c}(v)$ of color $c$ from $v$ to $a_{c}$. The three paths $P_{r}(v), P_{g}(v), P_{b}(v)$ pairwise only share the vertex $v$. The three paths of $v$ partition the interior of $G$ into 3 regions. For $\left\{c_{1}, c_{2}, c_{3}\right\}=\{r, g, b\}$ we let $R_{c_{1}}(v)$ be the region bounded by $P_{c_{2}}$ and $P_{c_{3}}$. With $v$ we associate the region vector $\left(v_{r}, v_{g}, v_{b}\right)$ where $v_{c}$ is the number of faces of $G$ in region $R_{c}(v)$. Fig. 14 illustrates regions and region vectors.

Let $\mathcal{V}=\left\{\left(v_{r}, v_{g}, v_{b}\right): v \in V(G)\right\}$ be the generating set for an orthogonal surface $\mathfrak{S}$. In slight abuse of notation we identify region vectors with their corresponding vertices and say that $\mathfrak{S}$ is generated by $V(G)$. The minima of $\mathfrak{S}$ are the vertices of $G$. Moreover, $\mathfrak{S}$ supports the Schnyder wood $S$ in the sense that every outgoing edge at $v$ in $S$ corresponds to an edge of the skeleton of $\mathfrak{S}$ such that the direction of the skeleton edge is given by the color of the edge. In fact from the clockwise order of directions of skeleton edges at minima, saddle points and maxima it can be concluded that the skeleton $G_{\mathfrak{S}}$ of $\mathfrak{S}$ is $T^{*}$. With Proposition 1 we obtain a Plattenbau representation of $T$. 


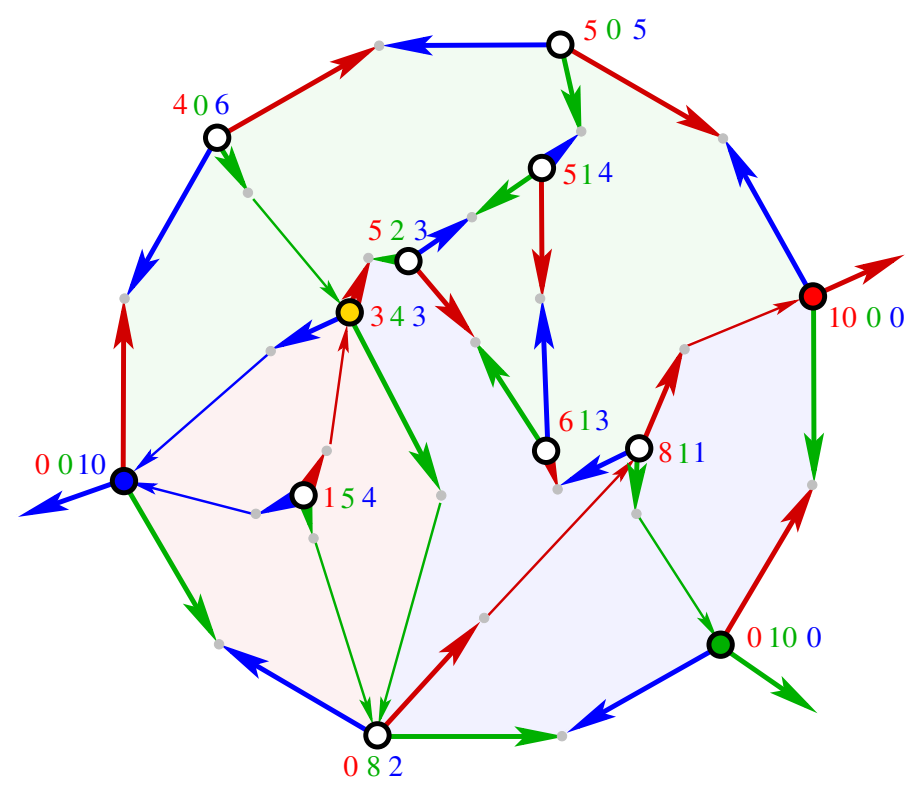

Fig. 15. A Schnyder wood with a shading indicating the regions of the yellow vertex. Vertices are labeled with their region vector.

Since the set of $\alpha$-orientations of a fixed planar graph carries the structure of a distributive lattice 9], and we have shown a correspondence of such a set with the orthogonal surfaces with given skeleton, we obtain:

Corollary 1. Let $G$ be a planar cubic bipartite graph with specified vertex $v_{\infty}$. The set of orthogonal surfaces with skeleton $G$ and vertex $v_{\infty}$ at infinity carries a distributive lattice structure.

\subsection{Plattenbau Representations in the Presence of Babets}

Let $T$ be a 3-colorable triangulation suppose that $T$ contains babets. Being separating triangles babets can be nested, let $\mathcal{B}$ be the family of basic babets of $T$, i.e., of babets which are not contained in the interior of another babet. Let $T_{\Delta}$ be the triangulation obtained from $T$ by cleaning all the babets, i.e., removing the interior vertices and their incident edges from all babets $B \in \mathcal{B}$. Clearly $T_{\Delta}$ is 3-colorable and babet-free. Triangles which have been babets are black. With the method from the previous subsection we get an orthogonal surface $\mathfrak{S}_{\Delta}$ for $T_{\Delta}$. In this representation triangles which have been babets correspond to saddle points. For later reference let $u_{B}$ be the saddle point corresponding to $B \in \mathcal{B}$.

For each babet $B \in \mathcal{B}$ let $T_{B}$ the inside triangulation of $B$ in $T$. Clearly, $T_{B}$ is 3-colorable, hence, its triangles can be colored black and white with the outer face being black (note that this coloring of $T_{B}$ is obtained from the coloring of 


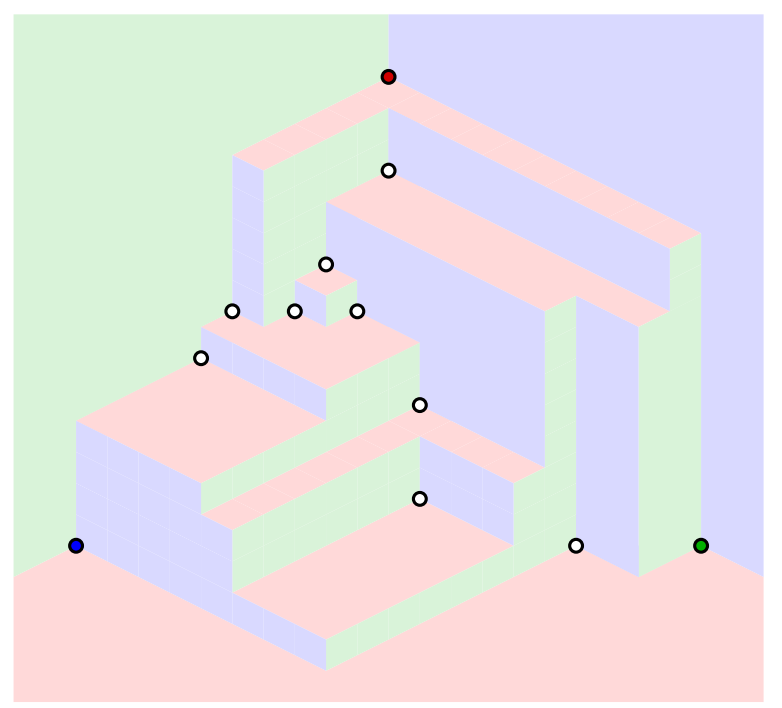

Fig. 16. The orthogonal surface obtained from the region vectors given in Fig. 15

triangles in $T$ by exchanging black and white). Assuming that $T_{B}$ has no babet we obtain an orthogonal surface $\mathfrak{S}_{B}$ for $T_{B}$.

The construction of the orthogonal surface $\mathfrak{S}_{B}$ works with the assumption that the vertices of the outer face, i.e., of the triangle $B$ are colored $r, g, b$ in clockwise order. The same assumption for the full triangulation $T$ implies that the vertices of $B$ are colored $r, b, g$ in clockwise order.

The goal is to patch $\mathfrak{S}_{B}$ at the saddle point $u_{B}$ to $\mathfrak{S}_{\Delta}$ so that the flats corresponding to a vertex of $B$ in the two orthogonal surfaces are coplanar. Let $f_{r}, f_{g}$, and $f_{b}$ be the red, green and blue flat at $u_{B}$ in $\mathfrak{S}_{\Delta}$, they represent the vertices $v_{r}, v_{g}, v_{b}$ of $B$ with their color in $T$. At $u_{B}$ exactly one of the three flats has a concave angle, we assume that this flat is $f_{r}$, the other cases are completely symmetric. The point $u_{B}$ is an interior point of the rectangle $R=R\left(f_{r}\right)$ spanned by the extreme points of $f_{r}$. The point $u_{B}$ is the apex of a convex corner whose sides coincide locally with $R \backslash f_{r}, f_{g}$ and $f_{b}$. In this corner we see the colors of the flats in clockwise order as $r, b, g$. Hence, we can patch and appropriately scaled down copy of $\mathfrak{S}_{B}$ into this corner such that the red outer flat of $\mathfrak{S}_{B}$ becomes part of $R \backslash f_{r}$, while the green outer flat of $\mathfrak{S}_{B}$ becomes part of $f_{b}$ and the blue outer flat of $\mathfrak{S}_{B}$ becomes part of $f_{g}$. With the technique of Proposition 1 we obtain a rectangle contact representation of the subgraph of $T$ induced by all the vertices of $T$ which are represented by flats of $\mathfrak{S}_{\Delta}$ and $\mathfrak{S}_{B}$. See Fig. 17 for an illustration.

Repeating the procedure for further babets in $\mathcal{B}$ and for babets which may occur in triangulations $T_{B}$ we eventually obtain an rectangle contact representation of $T$. This completes the proof of Theorem 1. 


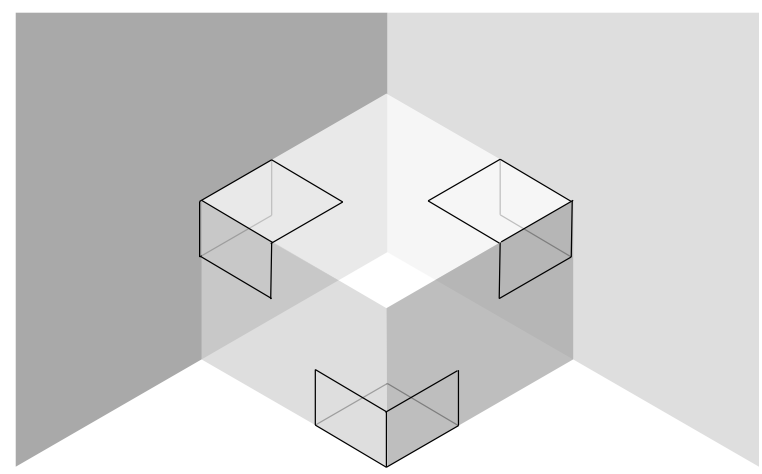

Fig. 17. The different places to patch an orthogonal surfaces into the saddle points of another orthogonal surface..

\subsection{Comments on Related Work}

Eppstein and Mumford 6] study orthogonal polytopes and their graphs.

They define corner polyhedra as polytopes obtained from what we call an orthogonal surface by restricting the surface to the bounded flats and connecting their boundary to the origin $\mathbf{0}=(0,0,0)$, this replaces the 3 unbounded flats of an orthogonal surface by three flats closing the polytope. Eppstein and Mumford show that the skeleton graphs of corner polyhedra are exactly the cubic bipartite 3 -connected graphs with the property that every separating triangle of the planar dual graph has the same parity. This is equivalent to our characterization of these graphs as duals of 3-colorable triangulations with admit a choice of the outer face such that there are no babets, see Proposition 2 .

A major part of their proof is devoted to the construction of a rooted cycle cover, respectively, to the investigation of necessary and sufficient conditions for the existence of such a cycle cover. The proof is based on a set of operations that allow any 4-connected Eulerian triangulation to be reduced to smaller ones. In contrast show the equivalent existence of an $\alpha$-orientation with a counting argument. Given a cycle cover Eppstein and Mumford provide a construction an appropriate orthogonal surface from the combinatorial data by combining the coordinates obtained from plane drawings of three orthogonal projections. In contrast we refer to the established theory of Schnyder woods and their relation to orthogonal structures to get the results.

As a more general class than corner polyhedra Eppstein and Mumford define $x y z$ polyhedra as orthogonal polytopes with the property that each axis-parallel line through a vertex contains exactly one other vertex. They characterize the skeletons of as cubic bipartite 3-connected graphs, i.e., as the duals of 3-colorable triangulations. Modulo the 'reflection' of the three outer flats this correspond to our Theorem 1. The main step in the proof is the gluing of an orthogonal surface into a corner of another orthogonal surface, see Section 3.2 and also Fig. 29 in 6 . 
A recent paper of Gonçalves 14 can be used as a basis for yet another proof of Theorem 1, i.e., a proof of the characterization of xyz polyhedra. Gonçalves uses a system of linear equations to construct a TC-scheme for a given 3-colorable triangulation. The TC-scheme comes very close to a segment contact representation with segments of 3 slopes for the input graph, however, there can be degeneracies: segments may degenerate to points (this relates to babets) and segments ending on two sides of another segment may have coinciding endpoints. The TC-scheme can be transformed into an orthogonal surface. First adjust the directions to have slopes $0,+\frac{\pi}{3}$, and $-\frac{\pi}{3}$, then add an orthogonal peak in each gray triangl $e^{6}$ and an orthogonal valley in each white triangle, and extend the outer flats. This yields an orthogonal surface. If there are no degeneracies the orthogonal surface properly represents the triangulation via flat contacts. Degeneracies of the TC-scheme translate into corners of degree 6 in the orthogonal surface, they can be resolved by shifting flats (c.f. 12 for details on flat shifting). Finally as in the other two approaches babets have to be recovered by patching their orthogonal surface into corners of the surface.
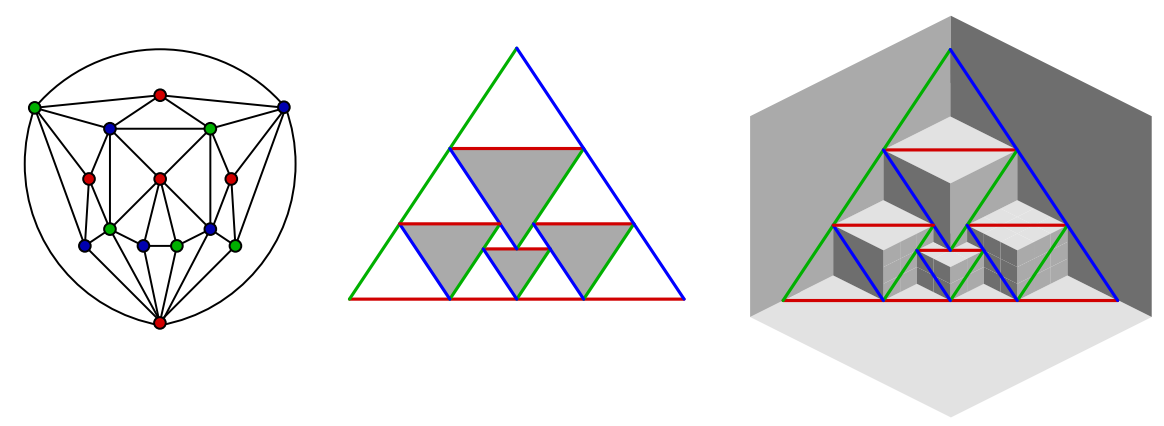

Fig. 18. A 3-colored triangulation, a TC-scheme of the triangulation and the corresponding orthogonal surface.

A nice aspect of this approach is that the partition of white triangles of the triangulation into peak and valley triangles is done by solving the linear system, no need of computing an $\alpha$-orientation or a cycle cover for this task.

\section{Proper Boxed Plattenbauten and Octahedrations}

In this section we characterize the touching graphs of proper boxed Plattenbauten, that is, we prove Theorem 2 .

First, in any proper Plattenbau any two touching rectangles $R, R^{\prime}$ have a proper contact, i.e., a boundary edge of one rectangle, say $R$, is completely

\footnotetext{
${ }^{6}$ This refers to the two color classes of the triangles of the TC-scheme, not to the two classes of triangles of the original triangulation.
} 
contained in the other rectangle $R^{\prime}$. We denote this as $R \rightarrow R^{\prime}$ and remark that this orientation has already been used in the proof of Observation 2.

Secondly, in any proper boxed Plattenbau $\mathcal{R}$ there are six rectangles that are incident to the unbounded region. We refer to them as outer rectangles and to the six corresponding vertices in the touching graph $G$ for $\mathcal{R}$ as the outer vertices. The corners incident to three outer rectangles are the outer corners, and the inner regions/cells of $\mathcal{R}$ will be called rooms.

Whenever we have specified some vertices of a graph to be outer vertices, this defines inner vertices, outer edges, and inner edges as follows: The inner vertices are exactly the vertices that are not outer vertices; the outer edges are those between two outer vertices; the inner edges are those with at least one inner vertex as endpoint. We shall use these notions for a Plattenbau graph, as well as for some planar quadrangulations we encounter along the way.

Let us start with the necessity of Items (P1) to (P4) in Theorem 2

Proposition 3. Every touching graph of a proper boxed Plattenbau satisfies Items (P1) to (P4) in Theorem 2.

Proof. Item (P1) follows directly from the definition of boxed Plattenbauten. Also, for Item (P2) simply orient each edge towards the end-vertex whose rectangle has interior points in the intersection. This way, any edge between two outer vertices is bidirected, which is in accordance with Item (P2). Now, Item (P3) follows from Observation 1] Item 2 together with the fact that edge-maximal planar bipartite graphs are quadrangulations. Indeed, if the rectangles on one side of a given rectangle would not induce a quadrangulation, then there would be a rectangle with a free edge and $\mathcal{R}$ would not be boxed. Let us finally argue Item (P4), The common neighbors of two vertices $u, v$ lie on the circle that is the intersection of the spheres with centers $S Q(u)$ and $S Q(v)$, respectively. Since $u$ and $v$ are on different sides of the intersection, $u, v$ see the vertices on the circle in opposite order. See Fig. 19 for an illustration.
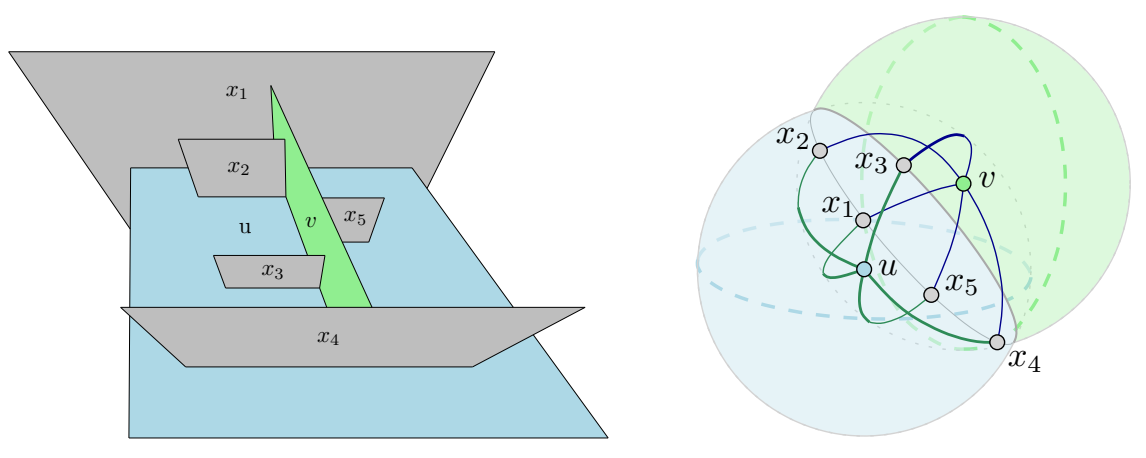

Fig. 19. Two rectangles (green and blue) and their common neighborhood. 
Next, we prove the sufficiency in Theorem 2, i.e., for every graph $G$ satisfying Items (P1) to (P4) we find a proper boxed Plattenbau with touching graph $G$.

Fix a graph $G=(V, E)$ with six outer vertices and edge orientation fulfilling Items (P1) to (P4). For each vertex $v \in V$ denote by $S Q(v)$ the spherical quadrangulation induced by $N(v)$ given in Item (P3). By Item (P3) the outneighbors of vertex $v$ induce a 4 -cycle in $S Q(v)$, which we call the equator $O_{v}$ of $S Q(v)$. The equator $O_{v}$ splits the spherical quadrangulation $S Q(v)$ into two hemispheres, each being a plane embedded quadrangulation with outer face $O_{v}$ with the property that each vertex of $S Q(v)-O_{v}$ is contained in exactly one hemisphere. The vertices of $O_{v}$ are the outer vertices of either hemisphere. Note that one hemisphere (or even both) may be trivial, namely when the equator bounds a face of $S Q(v)$.

We proceed with a number of claims.

Claim 2. In each hemisphere, each inner vertex has exactly two outgoing edges and no outer vertex has an outgoing inner edge.

Proof of Claim. Let $u$ be any inner vertex of a hemisphere of $S Q(v)$. We shall first show that $u$ has at least two out-neighbors in that hemisphere. We have $u \in N(v)-O_{v}$, i.e., $u$ is a neighbor of $v$ but not an out-neighbor. Hence, the edge $u v$ is directed from $u$ to $v$ and $v$ lies on the equator $O_{u}$ of $S Q(u)$. As such, $v$ has two neighbors $w_{1}, w_{2}$ on $O_{u}$, i.e., $w_{1}, w_{2} \in O_{u} \cap N(v)$. It follows that $w_{1}, w_{2}$ are out-neighbors of $u$, since $w_{1}, w_{2} \in O_{u}$, and $w_{1}, w_{2} \in S Q(v)$, since $w_{1}, w_{2} \in N(v)$. In other words, $u$ has at least two out-neighbors in $S Q(v)$ and, by planarity of $S Q(v)$, these are in the same hemisphere as $u$, as desired.

Finally, each hemisphere of $v$ is a plane quadrangulation with outer 4-cycle $O_{v}$, and as such has exactly $2 k$ inner edges for $k$ inner vertices. As each inner vertex has at least two outgoing edges, the edge count gives that each inner vertex has exactly two outgoing edges. Moreover, each inner edge is outgoing at some inner vertex, i.e., no outer vertex of the hemisphere has an outgoing inner edge.

Each equator edge of $S Q(v)$ induces together with $v$ a triangle in $G$, and we call these four triangles the equator triangles of $v$.

Claim 3. Every triangle in $G$ is an equator triangle.

Proof of Claim. Let $\Delta$ be a triangle in $G$ with vertices $u, v, w$. First, we shall show that $\Delta$ is not oriented as a directed cycle, i.e., has a vertex of out-degree two in $\Delta$. Without loss of generality let $u v$ be directed from $u$ to $v$. If $u w$ is directed from $u$ to $w$, we are done. So assume that $u w$ is directed from $w$ to $u$. Then $u \in N(v)-O_{v}$ is an inner vertex of a hemisphere of $S Q(v)$. Moreover $w \in N(v)$ has an outgoing edge to $u$, and Claim 2 implies that $w$ is an inner vertex of the same hemisphere of $S Q(v)$. In particular, $v w$ is directed from $w$ to $v$ and $w$ has out-degree two in $\Delta$, as desired.

Now let $a$ be the vertex in $\Delta$ with out-degree two and $b, c$ be its out-neighbors in $\Delta$. Then $b$ and $c$ lie on the equator of $a$ and are connected by an edge $b c$ in $G$. Thus $\Delta$ is an equator triangle. 
Clearly, a vertex $w$ forms a triangle with two vertices $u$ and $v$ if and only if $u v$ is an edge and $w$ is a common neighbor of $u$ and $v$. Equivalently, $w$ is adjacent to $v$ in $S Q(u)$, which in turn is equivalent to $w$ being adjacent to $u$ in $S Q(v)$. Hence, the set $N(u) \cap N(v)$ of all common neighbors (and thus also the set of all triangles sharing edge $u v$ ) is endowed with the clockwise cyclic ordering around $v$ in $S Q(u)$, as well as with the clockwise cyclic ordering around $u$ in $S Q(v)$. By Item (P4) these two cyclic orderings are reversals of each other.

Let us define for a triangle $\Delta$ in $G$ with vertices $u, v, w$ the two sides of $\Delta$ as the two cyclic permutations of $u, v, w$, which we denote by $[u, v, w]$ and $[u, w, v]$. So triangle $\Delta$ has the two sides $[u, v, w]=[v, w, u]=[w, u, v]$ and $[u, w, v]=$ $[w, v, u]=[v, u, w]$. We define a binary relation $\sim$ on the set of all sides of triangles in $G$ as follows.

$$
[u, v, a] \sim[v, u, b] \text { if }\left\{\begin{array}{l}
a \text { comes immediately before } b \\
\text { in the clockwise cyclic ordering } \\
\text { of } N(u) \cap N(v) \text { around } v \text { in } S Q(u)
\end{array}\right.
$$

Note that by (P4) $a$ comes immediately before $b$ in the clockwise ordering around $v$ if and only if $b$ comes immediately before $a$ in the clockwise ordering around $u$. Thus $[u, v, a] \sim[v, u, b]$ also implies $[v, u, b] \sim[u, v, a]$, i.e., $\sim$ is a symmetric relation and as such encodes an undirected graph $H$ on the sides of triangles.

Claim 4. Each connected component of $H$ is a cube. The corresponding subgraph in $G$ is an octahedron.

Proof of Claim. Consider any fixed vertex $[u, v, w]$ of $H$. Then $v w$ is an edge of $G$ contained in $S Q(u)$. As $S Q(u)$ is a quadrangulation, vertex $v$ has degree at least two in $S Q(u)$. Hence, there exists a unique vertex $a$ in $S Q(u)$ such that $[u, v, w] \sim[v, u, a]$ according to Eq. (1). Moreover, $a$ and $w$ are both neighbors of $v$ in $S Q(u)$ and hence non-adjacent in $G$, i.e., $a \in(N(u) \cap N(v))-N(w)$. Symmetrically, we find $b \in(N(w) \cap N(u))-N(v)$ with $[w, u, v] \sim[u, w, b]$ and $c \in(N(v) \cap N(w))-N(u)$ with $[v, w, u] \sim[w, v, c]$. It follows that $a, b, c$ are pairwise distinct vertices of $G$ and thus $[u, v, w]$ has degree exactly three in $H$.

Now recall that $v w$ is an edge of $G$ contained in $S Q(u)$. Consider the face $f$ in $S Q(u)$ for which $v$ comes immediately before $w$ in the clockwise ordering around $f$. Let $v, w, s, t$ be the clockwise ordering of vertices around $f$. Then, for example, $w$ comes immediately before $t$ in the clockwise cyclic ordering around $v$ in $S Q(u)$. Using Eq. (1), we have the following.

$$
\begin{aligned}
{[u, v, w] \sim[v, u, t]=[u, t, v] } & \sim[t, u, s] \\
= & {[u, s, t] \sim[s, u, w]=[u, w, s] \sim[w, u, v]=[u, v, w] }
\end{aligned}
$$

It follows that $t=a$ and $s=b$. I.e., the component of $H$ with vertex $[u, v, w]$ contains the four triangle sides $[u, v, w],[u, w, b],[u, b, a],[u, a, v]$ and these form a 4-cycle in $H$. As $v, w, a, b$ are pairwise distinct vertices in $G$, we have $[u, v, w] \neq$ $[u, b, a]$ and $[u, w, b] \nsim[u, a, v]$, meaning that the above 4 -cycle in $H$ is induced. 


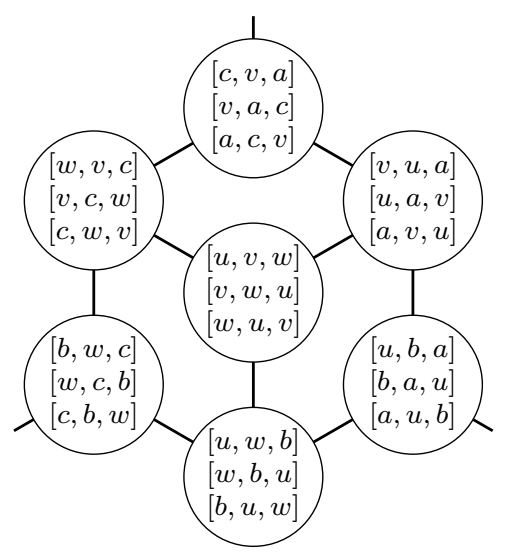

Fig. 20. The second neighborhood of vertex $[u, v, w]$ in the auxiliary graph $H$.

Repeating the same argument for $[v, w, u]$ in quadrangulation $S Q(v)$ and $[w, u, v]$ in quadrangulation $S Q(w)$, we get the induced 4-cycles

$$
\begin{aligned}
& {[v, w, u] \sim[w, v, c]=[v, c, w] \sim[c, v, a]} \\
& =[v, a, c] \sim[a, v, u]=[v, u, a] \sim[u, v, w]=[v, w, u]
\end{aligned}
$$

and

$$
\begin{aligned}
{[w, u, v] \sim[u, w, b]=[w, b, u] } & \sim[b, w, c] \\
& =[w, c, b] \sim[c, w, v]=[w, v, c] \sim[v, w, u]=[w, u, v]
\end{aligned}
$$

As $[u, v, w]=[v, w, u]=[w, u, v]$, these three induced 4-cycles in $H$ pairwise share exactly one edge, as shown in Fig. 20. For each of the three vertices $[a, c, v]$, $[b, a, u]$, and $[c, b, w]$, the third neighbor in $H$ is yet to be explored. However by symmetry, each of those vertices is also in exactly three induced 4-cycles, which implies that they have the same third neighbor: vertex $[a, b, c]=[b, c, a]=$ $[c, a, b]$.

Thus, the component of $H$ containing $[u, v, w]$ is a cube. The eight corresponding triangles in $G$ form an octahedron with vertex set $\{u, v, w, a, b, c\}$.

With Claim 4 we have identified a family of octahedra in $G$ such that each side of each triangle in $G$ is contained in exactly one octahedron. We call these octahedra the cells of $G$, as these correspond in the 2-dimensional case to the 4-cycles bounding faces of the quadrangulation. As Eq. (1) puts two triangle sides $[u, v, a]$ and $[v, u, b]$ into a common cell if and only if the edges $v a$ and $v b$ bound the same facial 4-cycle in $S Q(u)$, we obtain the following correspondence between the cells of $G$ and the faces in the spherical quadrangulations.

Claim 5. If $O \subseteq G$ is a cell of $G$ and $C$ is an induced 4-cycle in $O$, then $C$ bounds a face of $S Q(v)$ and a face of $S Q(u)$ for the two vertices $u, v \in O-C$. 
Conversely, if $C$ is a 4-cycle bounding a face of $S Q(v)$, then there is a cell $O$ of $G$ containing $\{v\} \cup V(C)$.

Having identified the cells, we can now construct a proper boxed Plattenbau for $G$ by identifying two opposite vertices in a particular cell, calling induction, and then splitting the rectangle corresponding to the identification vertex into two. The cells of $G$ will then correspond to the rooms in $\mathcal{R}$, except that one cell in $G$ will correspond to the unbounded region of $\mathcal{R}$ (which is not a room). To this end, we prove the following stronger statement:

Lemma 1. Let $G$ be a graph satisfying Items (P1) to (P4) and let $A, B, C$ be three outer vertices forming a triangle in $G$. Then there exists a proper boxed Plattenbau $\mathcal{R}$ whose touching graph is $G$ such that each of the following holds.

(I1) The six outer vertices of $G$ correspond to the outer rectangles of $\mathcal{R}$.

(I2) The cells of $G$ correspond to the rooms of $\mathcal{R}$, except for one cell that is formed by all six outer vertices.

(I3) For any two vertices $u, v$ with corresponding rectangles $R_{u}, R_{v}$ we have $u \rightarrow$ $v$ in the orientation of $G$ if and only if $R_{u} \cap R_{v}$ contains an edge of $R_{u}$.

(I4) For each vertex $v$ corresponding to a rectangle $R_{v}$, the rectangles touching $R_{v}$ come in the same spherical order as their corresponding vertices in $S Q(v)$.

Proof. We proceed by induction on the number of vertices in $G=(V, E)$. As the base case we have $|V|=6$ and $G \cong K_{2,2,2}$ is just an octahedron. In this case $G$ has exactly eight triangles and 16 sides of triangles. There are exactly two cells, each isomorphic to $G$. The desired Plattenbau $\mathcal{R}$ is given by the six sides of an axis-aligned cuboid in $\mathbb{R}^{3}$. It is easy to see that $\mathcal{R}$ has the required properties.

So let us assume that $|V|>6$, i.e., there is at least one inner vertex. Consider the three outer vertices $A, B, C$, which form a triangle in $G$. We shall first show that at least one of the quadrangulations $S Q(A), S Q(B), S Q(C)$ has an inner vertex. As $G$ is connected by Item (P1), there is an edge in $G$ from some inner vertex $v$ to some outer vertex $w$ with $w$ not necessarily in $\{A, B, C\}$. Then $v$ is an inner vertex of $S Q(w)$, i.e., one hemisphere of $S Q(w)$ is a plane quadrangulation with at least five vertices whose outer 4-cycle consists of four outer vertices of $G$. One edge of the outer 4-cycle has both endpoints in $\{A, B, C\}$ and, as there is some inner vertex, at least one of these two endpoints has an inner vertex as a neighbor.

So we may assume that $A$ has an inner neighbor, i.e., the non-trivial hemisphere $Q$ of $S Q(A)$ has an inner face $f$ that is bounded by the edge $B C$ and at least one inner vertex of $G$. Consider the cell $O$ of $G$ that contains all vertices on $f$, as given by Claim 5. Let the vertices of this octahedron $O$ be denoted by $A, B, C, a, b, c$ with the three pairs of non-adjacent vertices being $\{A, a\},\{B, b\}$, and $\{C, c\}$. Note that at least one of $a, b, c$ is in inner vertex of $G$ since $O$ includes an inner vertex of $Q$. In any case, vertices $a, b, c$ form a triangle in $G$ and each of the three vertices has outgoing edges to two of $A, B, C$. By Claim 3 , one of $a, b, c$, say $c$, has outgoing edges to the other two vertices ( $a$ and $b$ in this 
case). In particular, $c$ is an inner vertex, as otherwise $c$ has only outer vertices as out-neighbors (by Item (P2), contradicting that one of $a, b, c$ is an inner vertex.

Now we identify vertices $c$ and $C$ in $G$, denoting the resulting vertex by $\tilde{C}$ and the resulting graph by $\tilde{G}$. Each of $A, B, a, b$ is a neighbor of $c$ and $C$ in $G$. We remove double edges during the identification, so that in $\tilde{G}$ vertex $\tilde{C}$ is connected to each of $A, B, a, b$ with a single edge. In $\tilde{G}$ we choose the same six vertices except for $\tilde{C}$ replacing $C$ as the outer vertices. We claim that $\tilde{G}$ together with this choice of outer vertices has the properties in Items (P1) to (P4). Indeed Item (P1) holds because each neighbor $w$ of $\tilde{C}$ in $\tilde{G}$ that is not a neighbor of $C$ in $G$ has in $G$ an incoming edge to $c$. In particular, such $w$ is an inner vertex, as $c$ is an inner vertex. Hence the outer vertices in $\tilde{G}$ induce an octahedron. Connectivity of $\tilde{G}$ follows from connectivity of $G$.

For Item (P2) we first prove the following.

Claim 6. We have $N(c) \cap N(C)=\{A, B, a, b\}$.

Proof of Claim. Suppose for the sake of contradiction that $v$ is a common neighbor of $c$ and $C$ different from $A, B, a, b$. Then $C$ and $c$ are out-neighbors of $v$, i.e., $v$ is a non-equator vertex in $S Q(c)$ and $S Q(C)$. Let $w$ be an out-neighbor of $v$ different from $c, C$. Then, by Claim $2 w$ is contained in $S Q(c)$ and $S Q(C)$, too. If $w$ is neither on the equator of $S Q(c)$ nor on the equator of $S Q(C)$, we can repeat the argument with $w$ taking the role of $v$. Thus assume that $w$ is an out-neighbor of at least one of $c, C$. As out-neighbors of the outer vertex $C$ are outer vertices, it follows that $w$ is an out-neighbor of $c$, i.e., $w \in\{A, B, a, b\}$. By symmetry, assume that $w \in\{B, b\}$. Now consider the hemisphere $Q$ of $S Q(w)$ that contains $v$ as an inner vertex. As $A, B, C, a, b, c$ form a cell, the vertices $A, C, a, c$ form a quadrangular face in $Q$ by Claim 5 . Moreover, we know that $c$ has outgoing edges to $A$ and $a$, while $v$ has outgoing edges to $c$ and $C$, see Fig. 21 for an illustration of the situation on $S Q(w)$.

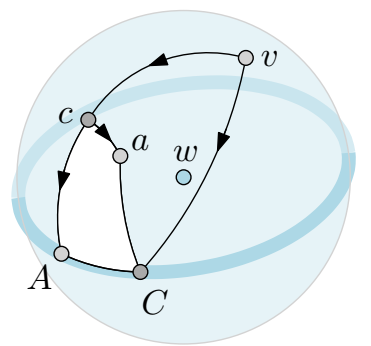

Fig. 21. The situation in Claim 6 that leads to a contradiction.

Vertices $a, A, c, C$ and $v$ induce a $K_{2,3}$ in $S Q(w)$. Since $a, A, c, C$ bound a face of the hemisphere $Q$ of $S Q(w)$, and $A, C$ are outer vertices of $G$ (hence outer vertices of $Q$ ), it follows that vertex $a$ lies inside the 4-cycle $K$ formed by $A, c, v, C$ in $Q$. As $Q$ is a quadrangulation, the 4 -cycle $K$ together with the 
vertices in its interior is as well a quadrangulation $J$ with outer face $K$. As such, $J$ has exactly $2|V(J)|-8$ inner edges on $|V(J)|-4$ inner vertices. One of these inner edges of $J$, namely the edge $c a$ is outgoing at the outer vertex $c$ of $J$. Hence at most $2|V(J)|-9$ inner edges of $J$ are outgoing at an inner vertex of $J$. But this is a contradiction to Claim 2, which states that each of the $|V(J)|-4$ inner vertices has exactly two outgoing edges in $Q$.

Claim 6 implies that the orientation of $G$ given by Item (P2) naturally induces an orientation of $\tilde{G}$ satisfying again Item (P2).

Claim 7. Item (P3) holds for $\tilde{G}$.

Proof of Claim. We shall show that for each vertex $v$ of $\tilde{G}$, the neighborhood of $v$ induces a spherical quadrangulation $S Q(v)$ in which the out-neighbors of $v$ form a 4-cycle. We distinguish different cases of $v$ and how $N(v)$ changes during the identification of $c$ and $C$. For $v=\tilde{C}, N(v)$ in $\tilde{G}$ is the union of $N(c)$ and $N(C)$ in $G$. As $A, B, C, a, b, c$ form a cell, $A, B, a, b$ form a face in both $S Q(c)$ and $S Q(C)$ by Claim 5. Moreover, $A, B, a, b$ are the equator in $S Q(c)$. Hence, the subgraph of $G$ induced by $N(v)=N(c) \cup N(C)$ can be obtained by pasting $S Q(c)$ into the face $A, B, a, b$ of $S Q(C)$. This is a quadrangulation and the outneighbors of $\tilde{C}$ are the same as for $C$, i.e., induce a 4-cycle. For $v \in\{A, B, a, b\}$, identifying $C$ and $c$ corresponds to merging opposite vertices of a face $f$ in $S Q(v)$. As $N(c) \cap N(C)=\{A, B, a, b\}$ by Claim 6, in $S Q(v)$ vertices $c$ and $C$ have no common neighbor outside of $f$. Thus the identification of $C$ and $C$ in $S Q(v)$ preserves the property of being a quadrangulation and does not affect the equator. For $v \notin\{\tilde{C}, A, B, a, b\}$ the neighborhood of $v$ does not change, except for possibly renaming $c$ or $C$ to $\tilde{C}$. This shows that $\tilde{G}$ satisfies Item (P3).

Claim 8. Item (P4) holds for $\tilde{G}$.

Proof of Claim. Recall that during the identification of $c$ and $C$, we changed the embedding of $S Q(v)$ only for $v \in\{\tilde{C}, A, B, a, b\}$. Thus we need to check only those edges in $\tilde{G}$ with at least one endpoint in $\{\tilde{C}, A, B, a, b\}$. First consider an edge $\tilde{C} v$ with $v \notin\{A, B, a, b\}$. If $v$ was a neighbor of $c$ in $G$, then in $S Q(\tilde{C})$ $v$ is embedded inside the quadrangle $A, B, a, b$. If $v$ was a neighbor of $C$ in $G$, then in $S Q(\tilde{C}) v$ is embedded outside the quadrangle $A, B, a, b$. By planarity of $S Q(\tilde{C})$, there is no edge between these two types of vertices. Thus the common neighborhood of $\tilde{C}$ and any $v \notin\{A, B, a, b\}$ coincides with either $N(c) \cap N(v)$ or $N(C) \cap N(v)$ in $G$. Say $v$ was a neighbor of $c$. As the clockwise cyclic ordering around $v$ in $S Q(\tilde{C})$ is the same as in $S Q(c)$, and the clockwise ordering around $\tilde{C}$ in $S Q(v)$ is the same as that around $c$ in $S Q(v)$ before, Item (P4) is satisfied here. The case of an edge $\tilde{C} v$ with $v \in\{A, B, a, b\}$ and the other cases are similar. $\triangle$

Up to now we have shown that the graph $\tilde{G}$ obtained from $G$ by identifying $c$ and $C$ satisfies Items (P1) to (P4). Thus, by induction there is a proper boxed Plattenbau $\mathcal{R}^{\prime}$ whose touching graph is $\tilde{G}$ such that Items (I1) to (I4) hold. 
In particular the rectangles $R_{A}, R_{B}, R_{a}, R_{b}$ for the 4-cycle $A, B, a, b$ in $S Q(\tilde{C})$ enclose a rectangular region in one corner of the rectangle $R$ for $\tilde{C}$ (possibly entire $R$ ). We alter $\mathcal{R}$ by shortening all rectangles touching $R$ inside this region by the same small amount $\varepsilon>0$ and introducing a new rectangle $R_{c}$ for $c$ parallel to $R$ at distance $\varepsilon$, touching all shortened rectangles and the rectangles $R_{A}, R_{B}, R_{a}, R_{b}$. Secondly, we let $R$ be the rectangle for $C$. Then the resulting Plattenbau $\mathcal{R}$ represents $G$ as its touching graph, and Items (I1) to (I4) hold for $\mathcal{R}$.

Lemma 1 shows the sufficiency of Items (P1) to (P4) The necessity is given in Proposition 3. Together this proves Theorem 2 and concludes this section.

\section{Conclusions}

Touching graphs of proper boxed Plattenbauten are natural generalizations of quadrangulations from the plane to space. As we have shown these graphs are octahedrations of 3-space. These graphs can be seen as a novel way of going beyond planarity. A question in this spirit was asked by Jean Cardinal at the Order \& Geometry Workshop at Gutowy Palace in 2016: What is the 3-dimensional analogue of Baxter permutations? This is based on Baxter permutations being in bijection with boxed arrangements of axis-parallel segments in $\mathbb{R}^{2}[11$.

A continuation of this project to higher dimensions would be to consider proper boxed Plattenbauten in $\mathbb{R}^{d}$ and study the resulting touching graphs as generalizations of plane quadrangulations to arbitrary dimensions.

Considering the intersection graph $I_{\mathcal{R}}$ instead of the touching graph of a Plattenbau, yields a very different graph class. Its plane analogue is known as $\mathrm{B}_{0}$-CPG graphs, see [5]. For example, every 4-connected triangulation has a rectangle contact representation in $\mathbb{R}^{2}$, see $\left[18,19,23,26,28\right.$. Also $K_{12}$ is the intersection graph of the Plattenbau $\mathcal{R}$ consisting of the twelve axis-parallel unit squares in $\mathbb{R}^{3}$ that have a corner on the origin.

\section{References}

1. Philip L Bowers. Circle packing: a personal reminiscence. The Best Writing on Mathematics, pages 330-345, 2010.

2. Adam L Buchsbaum, Emden R Gansner, Cecilia M Procopiuc, and Suresh Venkatasubramanian. Rectangular layouts and contact graphs. ACM Transactions on Algorithms (TALG), 4(1):1-28, 2008.

3. L.Sunil Chandran, Rogers Mathew, and Naveen Sivadasan. Boxicity of line graphs. Discr. Math., 311(21):2359-2367, 2011.

4. Hubert De Fraysseix and P Ossona de Mendez. On topological aspects of orientations. Discrete Mathematics, 229(1-3):57-72, 2001.

5. Zakir Deniz, Esther Galby, Andrea Munaro, and Bernard Ries. On contact graphs of paths on a grid. In Therese Biedl and Andreas Kerren, editors, Graph Drawing and Network Visualization, pages 317-330, Cham, 2018. Springer International Publishing. 
6. David Eppstein and Elena Mumford. Steinitz theorems for simple orthogonal polyhedra. Jour. of Comp. Geom., 5(1):179-244, 2014.

7. Stefan Felsner. Convex drawings of planar graphs and the order dimension of 3-polytopes. Order, 18:19-37, 2001.

8. Stefan Felsner. Geometric Graphs and Arrangements. Vieweg Verlag, 2004.

9. Stefan Felsner. Lattice structures from planar graphs. Electr. J. of Comb., 11(R15):24p., 2004.

10. Stefan Felsner. Rectangle and square representations of planar graphs. In Thirty Essays on Geometric Graph Theory, pages 213-248. Springer, 2013.

11. Stefan Felsner, Éric Fusy, Marc Noy, and David Orden. Bijections for Baxter families and related objects. J. Comb. Theory, Ser. A, 118(3):993-1020, 2011.

12. Stefan Felsner and Florian Zickfeld. Schnyder woods and orthogonal surfaces. Discr. and Comp. Geom., 40:103-126, 2008.

13. Éric Fusy. Combinatoire des cartes planaires et applications algorithmiques. PhD thesis, Citeseer, 2007.

14. Daniel Gonçalves. 3-colorable planar graphs have an intersection segment representation using 3 slopes. In Proc. WG 2019., volume 11789 of $L N C S$, pages 351-363. Springer, 2019.

15. Terja Hansen and Herbert Scarf. The Computation of Economic Equilibria, volume 24 of Cowles Foundation Monograph. Yale Univ. Press, 1973.

16. I Ben-Arroyo Hartman, Ilan Newman, and Ran Ziv. On grid intersection graphs. Discrete Mathematics, 87(1):41-52, 1991.

17. Paul Koebe. Kontaktprobleme der konformen Abbildung. Hirzel, 1936.

18. Krzysztof Koźmiński and Edwin Kinnen. Rectangular duals of planar graphs. Networks, 15(2):145-157, 1985.

19. Sany M Leinwand and Yen-Tai Lai. An algorithm for building rectangular floorplans. In 21st Design Automation Conference Proceedings, pages 663-664. IEEE, 1984.

20. Ezra Miller. Planar graphs as minimal resolutions of trivariate monomial ideals. Documenta Math., 7:43-90, 2002.

21. Ezra Miller and Bernd Sturmfels. Combinatorial Commutative Algebra. Graduate Texts in Mathematics. Springer, 2004

22. János Pach, $\mathrm{H}$ de Fraysseix, and $\mathrm{P}$ de Mendez. Representation of planar graphs by segments. Technical report, North-Holland, 1994.

23. Pierre Rosenstiehl and Robert E Tarjan. Rectilinear planar layouts and bipolar orientations of planar graphs. Discrete \& Computational Geometry, 1(4):343-353, 1986.

24. Kenneth Stephenson. Introduction to circle packing: The theory of discrete analytic functions. Cambridge University Press, 2005.

25. Roberto Tamassia and Ioannis G Tollis. A unified approach to visibility representations of planar graphs. Discrete \& Computational Geometry, 1(4):321-341, 1986.

26. Carsten Thomassen. Interval representations of planar graphs. Journal of Combinatorial Theory, Series B, 40(1):9-20, 1986.

27. Torsten Ueckerdt. Geometric Representations of Graphs with Low Polygonal Complexity. Doctoral thesis, Technische Universität Berlin, Fakultät II - Mathematik und Naturwissenschaften, Berlin, 2012. URL: http://dx.doi.org/10.14279/ depositonce-3190

28. Peter Ungar. On diagrams representing graphs. Journal of the London Mathematical Society, 28:336-342, 1953. 